\title{
Dynamic model-based dose calculations of permanent implant prostate brachytherapy
}

\author{
by
}

\section{Luke P. McCooeye}

A thesis submitted to the Faculty of Graduate and Post Doctoral Affairs in partial fulfillment of the requirements for the degree of

\section{Master of Science}

in

\author{
Physics \\ Specialization in Medical Physics \\ Ottawa-Carleton Institute for Physics \\ Department of Physics \\ Carleton University \\ Ottawa, Ontario
}

(c) 2019

Luke P. McCooeye 


\section{Abstract}

This thesis presents the development of a framework for performing dynamic modelbased dose calculations in target organs exhibiting geometry changes during permanent implant brachytherapy. The framework is applied to investigate the dosimetric

effect of edema during ${ }^{125} \mathrm{I}$ prostate brachytherapy. Dynamic dose distributions are accumulated from mapped dose distributions calculated using Monte Carlo (MC) simulations on treatment geometries predicted from post-implant images and clinicallyobserved models of edema. Ten unique edema resolution models are implemented for 120 patients. Clinical AAPM TG-43 and static MC dose calculations overestimate $\mathrm{D}_{90}$ values compared to dynamically-calculated dose distributions. These overestimations range from $5 \%$ to $>30 \%$ depending on edema model and patient parameters, e.g., the presence of intraprostatic calcifications. Edema should be considered for accurate dose calculations of permanent implant prostate brachytherapy treatments. 


\section{Acknowledgments}

I would like to thank my supervisors Dr Rowan Thomson and Dr Emily Heath for all of the opportunities and guidance they have given me, and for being exemplary in their roles as supervisors. It has been an incredible experience and a pleasure to work with them.

I owe a debt of gratitude to the family and friends of Robert L. Clarke for making the transition into graduate studies a little easier with the Robert L. Clarke Graduate Scholarship.

To my comrades in the Carleton Laboratory for Radiotherapy Physics, for always being quick with a joke and even quicker to lend a helping hand. It would not have been the same without you.

To my partner Shannon Young, for her infinite patience with me during my studies, thank you for taking this ride with me.

Finally, to my grandmother Audrey, to whom I owe more than I could ever write,

I dedicate this to you. 


\section{Statement of Originality}

This thesis represents the research undertaken by the author during enrollment at Carleton University in the MSc program in Medical Physics.

Dr Emily Heath and Dr Rowan Thomson made contributions to the entirety of this research through study design, supervision and guidance, and manuscript revisions. Dr Luc Beaulieu contributed the initial patient database, expertise and feedback. Dr Nelson Miksys contributed 30-day post-implant virtual patient models (VPMs) compatible with the used software. Gavin Hurd did initial work on creating intermediate patient geometries. Software for extracting dosimetric quantities was adapted from 3ddose_tools, a program by Martin Martinov.

Work of the author included further development of the software for creating intermediate VPMs including the handling of rigid bodies within the geometry, creating the dose accumulation scheme and software, adapting and correcting existing software where required, and validation of all of the forementioned as well as performing calculations and analyzing the results.

Material in this thesis was presented by the author at the following conferences.

- L. McCooeye, G. Hurd, L. Beaulieu, E. Heath and R.M. Thomson. Monte Carlo dose calculations modelling edema resolution in permanent implant prostate brachytherapy

Poster presentation (\#241) at the CARO-COMP-CAMRT Joint Scientific Meeting. September 12-15, 2018 in Montreal, QC

- L. McCooeye, G. Hurd, L. Beaulieu, R.M. Thomson and E. Heath Dynamic model-based dosimetry of permanent implant brachytherapy Oral presentation at International Conference on Monte Carlo Techniques for Medical Applications, June 19-21, 2019 in Montreal, QC 


\title{
List of Acronyms and Notation
}

\author{
AAPM American Association of Physicists in Medicine \\ ABS American Brachytherapy Society \\ CTV Clinical Treatment Volume \\ D $\quad$ Dose \\ $\mathrm{D}_{\#} \quad$ minimum dose in hottest \#\% of the target volume \\ DVH Dose-Volume Histogram \\ d 3D dose distribution \\ $d_{\rightarrow \text { ref }} \quad 3 \mathrm{D}$ dose distribution mapped to the reference geometry \\ $d_{\text {ref }} \quad 3 \mathrm{D}$ dose distribution calculated using reference Monte Carlo conditions \\ $d_{\text {ref }}^{a c c} \quad 3 \mathrm{D}$ dose distribution of accumulated dose \\ EBRT External Beam Radiation Therapy \\ HDR High Dose Rate \\ IC Intraprostatic Calcification \\ \% IC amount of Intraprostatic Calcification as \% by volume of prostate \\ LDR Low Dose Rate \\ MBDCA Model-Based Dose Calculation Algorithm \\ MC Monte Carlo \\ NC No Calcification \\ NIST National Institute of Standards and Technology \\ OAR Organ At Risk \\ PIPB Permanent Implant Prostate Brachytherapy \\ $\mathrm{t}_{1 / 2}^{e} \quad$ edema resolution half-life \\ $\mathrm{t}_{1 / 2}^{s} \quad$ source half-life \\ TRUS TransRectal UltraSound \\ VPM Virtual Patient Model \\ $\mathrm{V}_{\#} \quad \%$ of target volume receiving dose $>\#$ \\ $\Delta \quad$ edema magnitude (volume as fraction of reference)




\section{Contents}

$\begin{array}{ll}\text { Abstract } & \text { ii }\end{array}$

Acknowledgments

Statement of Originality iv

List of Acronyms and Notation $\quad$ v

Table of Contents vi

List of Tables $\quad$ viii

List of Figures $\quad$ ix

1 Introduction 1

1.1 Radiotherapy . . . . . . . . . . . . . . . . . . . . . 1

1.2 Brachytherapy . . . . . . . . . . . . . . . . . 2

1.3 Permanent implant prostate brachytherapy . . . . . . . . . 5

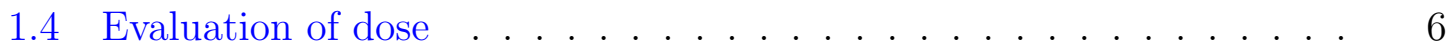

1.4 .1 TG-43 formalism . . . . . . . . . . . . . . . . 9

1.4 .2 Dose metrics . . . . . . . . . . . . . . . . . . 11

1.4 .3 Implant criteria . . . . . . . . . . . . . . . . . 11

1.4.4 Challenges in brachytherapy dose calculations . . . . . . . 12

1.4.5 Model-based dose calculation algorithms . . . . . . . . 16

1.5 Thesis purpose $\ldots \ldots \ldots \ldots \ldots \ldots$

1.6 Thesis outline $\ldots \ldots \ldots \ldots \ldots$ 
2 Methods $\quad 22$

2.1 Patient data . . . . . . . . . . . . . . . . . . 22

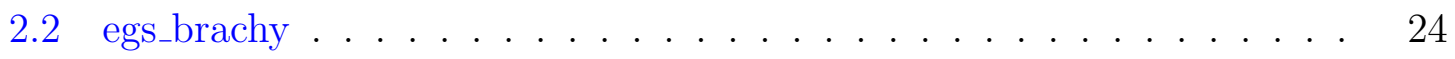

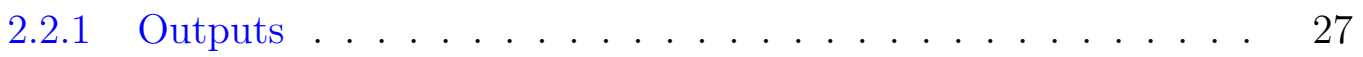

2.2.2 Comparison of egs_brachy to BrachyDose . . . . . . . . . . . . 27

2.2.3 Simulating TG-43 in egs_brachy . . . . . . . . . . . . . . . . . 29

2.3 dtransform . . . . . . . . . . . . . . . . . . . . . . . . . 29

2.4 Edema modelling process . . . . . . . . . . . . . . . . . 30

2.4.1 Generating intermediate geometries with edema . . . . . . . . 31

2.4 .2 Dose mapping . . . . . . . . . . . . . . . . . 37

2.4.3 Dose accumulation . . . . . . . . . . . . . . . . 37

2.4.4 Time-course discretization . . . . . . . . . . . . . . . . . 39

2.4.5 Dose evaluation ................... 41

2.4.6 Edema resolution models . . . . . . . . . . . . . . . 42

3 Results $\quad 44$

3.1 Time-course discretization . . . . . . . . . . . . . . . . 44

3.2 Analysis of dose mapping around a single seed . . . . . . . . . . . 46

3.3 Dose accumulation in example patients . . . . . . . . . . . . . 47

3.4 Dynamic MC compared to TG-43 . . . . . . . . . . . . 54

3.5 Dynamic MC compared to static MC . . . . . . . . . . 57

4 Discussion and conclusions $\quad 60$

4.1 Discussion . . . . . . . . . . . . . . . . . . . 60

4.2 Conclusions ........................ 65

$\begin{array}{ll}\text { References } & 66\end{array}$ 


\section{List of Tables}

1.1 Summary of radionuclides commonly used in brachytherapy . . . . . 4

1.2 Implant criteria . . . . . . . . . . . . . . . . . . . . . . . . 13

2.1 Assignment of tissue . . . . . . . . . . . . . . . . . . 24

2.2 Monte Carlo parameters (TG-268) . . . . . . . . . . . . . . 26

2.3 Models of edema resolution . . . . . . . . . . . . . . . . . 43

3.1 Effect of edema on $\mathrm{D}_{90}, \mathrm{D}_{99}, \mathrm{~V}_{100}$ and $\mathrm{V}_{200} \ldots \ldots \ldots$ 


\section{List of Figures}

1.1 Permanent implant prostate brachytherapy . . . . . . . . . . . . 5

1.2 Interaction cross sections . . . . . . . . . . . . . . . . 7

1.3 Electron transport . . . . . . . . . . . . . . . . . . . 10

1.4 Dose-volume histogram . . . . . . . . . . . . . . . . . . . 12

1.5 Mass-energy absorption coefficients . . . . . . . . . . . . . 16

$1.6{ }^{125} \mathrm{I}$ photon spectrum . . . . . . . . . . . . . . . . . . 17

2.1 PIPB procedure overview . . . . . . . . . . . . . . . 23

2.2 Calculated dose comparison with BrachyDose . . . . . . . . . . 28

2.3 Energy mapping . . . . . . . . . . . . . . . . . . . . . 31

2.4 Evaluating nominal density approximation . . . . . . . . . . . . 32

2.5 Resolving seed conflicts . . . . . . . . . . . . . . . . . . 36

2.6 Determining accumulation weights . . . . . . . . . 40

3.1 Time resolution sensitivity . . . . . . . . . . . . . . . . 45

3.2 Dose mapping validation . . . . . . . . . . . . . . . . . . . 46

3.3 Dose accumulation: $\mathrm{NC} \ldots \ldots \ldots \ldots$. . . . . . . . . . . . 48

3.4 Dose accumulation: $0.33 \% \mathrm{IC} \ldots \ldots . \ldots$. . . . . . . . . 50

3.5 Dose accumulation: $2.2 \% \mathrm{IC} \ldots \ldots \ldots$. . . . . . . . . . 51

3.6 Edema models have consistent effects relative to each other . . . . . . 53

3.7 Comparing $\mathrm{MC}$ to $\mathrm{TG}-43\left(\mathrm{D}_{90}\right) \ldots \ldots \ldots$. . . . . . . . 55

3.8 Comparing $\mathrm{MC}$ to $\mathrm{TG}-43\left(\mathrm{D}_{99}\right) \ldots \ldots \ldots$

3.9 Comparing dynamic $\mathrm{MC}$ to static $\mathrm{MC}\left(\mathrm{D}_{90}\right) \ldots \ldots . \ldots$

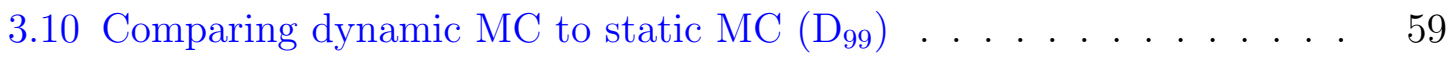




\section{Chapter 1}

\section{Introduction}

It is estimated that prostate cancer affects 1 in 7 Canadian men, accounting for roughly $20 \%$ of all new male cancer cases. ${ }^{1}$ There are several potential treatments (in-

cluding watchful-waiting, ${ }^{2}$ chemotherapy, ${ }^{3}$ and cryoablation $^{4}$ ) but the two standard treatment modalities are radical prostatectomy and radiotherapy. Each treatment has its advantages and disadvantages to be evaluated on a patient-by-patient basis. Radiotherapy is appropriate for many patients, either as a standalone treatment or as part of a combination therapy.

\section{$1.1 \quad$ Radiotherapy}

The principle of radiotherapy is to deliver a dose of ionizing radiation to the treatment target. The absorbed dose is the expectated value of the energy absorbed from radiation, $\left\langle E_{a b}\right\rangle$, per unit mass, $m$,

$$
D=\frac{\left\langle E_{a b}\right\rangle}{m} .
$$

Absorbed dose, or simply dose, is typically given in units of Gray (Gy), equivalent to one Joule of energy absorbed per kilogram of mass.

One mechanism of radiotherapy is to damage cellular DNA either directly (through energy transfer), or indirectly (through the creation of free radicals, reactive chemical species that interact with the DNA molecule). The likelihood of damage occurring 
increases with the dose. ${ }^{5}$ However, radiation-induced damage can occur not only in cancerous cells but also in otherwise healthy cells, causing side effects and secondary cancers. Consequently, the art and science of radiotherapy lies in finding the balance of delivering the maximum dose to cancerous tissues while limiting the dose to healthy tissue.

In addition to its targeted nature (in contrast to the whole-body effect of chemotherapy), radiation therapy can also benefit from the cellular biology of cancer. For most of the life of a cell the DNA within the nucleus is densely packed. During division the DNA must be unwound and is less protected from damage. ${ }^{6}$ Cancer cells proliferate by dividing more frequently than normal cells which means that DNA in a cancerous cell is more vulnerable to damage compared to a normal cell. Additionally, normal cells have various DNA repair mechanisms but in cancerous cells these repair mechanisms are often compromised. ${ }^{6}$ These two factors combined make cancerous cells more susceptible to radiation-induced DNA damage and boost the effectiveness of radiotherapy. ${ }^{5}$

Radiotherapy can be broadly separated into two categories. External beam radiation therapy (EBRT) is any treatment where the radiation source is external to the patient, such as the use of a linear accelerator. In contrast to EBRT, brachytherapy is any radiotherapy where the source is inside or on the patient.

\subsection{Brachytherapy}

Brachytherapy can take many practical forms depending on the location of the target. For sites near an external surface of the body, treatment can take the form of a radiation source placed superficially, as in the use of eye-plaque brachytherapy for the treatment of ocular tumours. ${ }^{7}$ Intracavitary insertion of a radiation source can be used for treatment of sites near a body cavity, such as gynecological cancers. ${ }^{8}$ 
Alternatively, radiation sources can be placed interstitially to treat sites that are located deeper in the body but not near a cavity, such as tumours in the breast $^{9}$ or prostate. ${ }^{10}$ In an interstitial treatment, multiple enclosed capsules, or 'seeds', containing a radiation source are implanted into the target tissue. Brachytherapy treatments are classified as temporary or permanent implants. Temporary implants can use either high dose rate $(\mathrm{HDR},>12 \mathrm{~Gy} / \text { hour at a range of } 1 \mathrm{~cm})^{11}$ or low dose rate $(\mathrm{LDR},<2 \mathrm{~Gy} / \text { hour at a range of } 1 \mathrm{~cm})^{11}$ sources, while permanent implants strictly use LDR sources. There is a medium dose rate that falls between LDR and HDR, but it is not used in prostate brachytherapy.

The choice of dose rate can be heavily weighted by the practical aspects of treatment delivery, but the dose rate can have a radiobiological effect. ${ }^{12}$ Tumour cells can retain some functionality in DNA repair mechanisms in addition to the ability to repopulate quickly, so there can be a dose rate effect where a dose delivered over a short period of time is more effective at causing irreparable damage than the same total dose over a longer period, ${ }^{13}$ particularly for a dose delivered at rates of $<1 \mathrm{~Gy} / \mathrm{h}^{12}$ However, an argument can be made in favour of lower dose rates as well because tumours often develop with poor vasculature and as a result many intratumour cells are poorly oxygenated, and hypoxia increases radioresistance. ${ }^{14}$ Delivering a dose more gradually allows for hypoxic cells to reoxygenate as well-oxygenated cells die off and oxygen availability increases, ${ }^{12}$ increasing the radiosensitivity of the remaining cells.

Table 1.1 summarizes photon-emitting radionuclides commonly used in brachytherapy. Characteristics of a good source can vary depending on the modality, but universally a high specific activity (activity per mass of radionuclide) is desirable to allow for small sources. For temporary implants the patient can be isolated during the treatment, so emissions with higher energy per photon are acceptable. In contrast, permanently implanted sources should have relatively low energy emissions so that very little radiation escapes from the patient. From a clinical standpoint a long 
Table 1.1: Summary of radionuclides commonly used in brachytherapy and their properties; half-lives and mean emitted photon energies. ${ }^{16}$

\begin{tabular}{lllc}
\hline Radionuclide & Use & Half-life & Mean photon energy $(\mathrm{keV})$ \\
\hline \hline${ }^{103} \mathrm{Pd}$ & Permanent & $17.0 \mathrm{~d}$ & 21 \\
${ }^{125} \mathrm{I}$ & Permanent & $59.4 \mathrm{~d}$ & 28 \\
${ }^{137} \mathrm{Cs}$ & Temporary & $30.0 \mathrm{y}$ & 660 \\
${ }^{192} \mathrm{Ir}$ & Temporary & $73.8 \mathrm{~d}$ & 355 \\
\hline
\end{tabular}

half-life is desirable for a temporary implant radionuclide so that it does not need to be replaced regularly. Shorter half-lives are preferred for permanent implants so that the dose is delivered at a sufficient rate for cell killing. ${ }^{15}$

Radionuclide brachytherapy sources encapsulate the radioactive material in a metal shell. A radioactive isotope of Iodine $\left({ }^{125} \mathrm{I}\right)$ is a frequently used radionuclide in permanent implant prostate brachytherapy (PIPB) treatments. The radioactive material and the capsule together comprise a 'seed'. Individual seed models vary in construction but a seed is generally cylindrical with approximate dimensions of 5 $\mathrm{mm}$ in length and $1 \mathrm{~mm}$ in diameter with the source distributed evenly within. In addition to the radionuclide used, the dose delivered by a treatment seed depends on the construction of the seed because the material of the shell can absorb and scatter radiation from the source, contributing to the spectrum of radiation leaving the seed. Shielding by the capsule is most pronounced where the seed has been welded shut at the ends.

Seeds can come assembled in 'strands', strings of multiple capsules connected by wire, or as 'loose' individual seeds. Stranded seeds are associated with reduced seed migration, ${ }^{17}$ but less conformal doses. ${ }^{18}$ There is no significant difference in outcomes between treatments using loose and stranded seeds. ${ }^{18}$ 


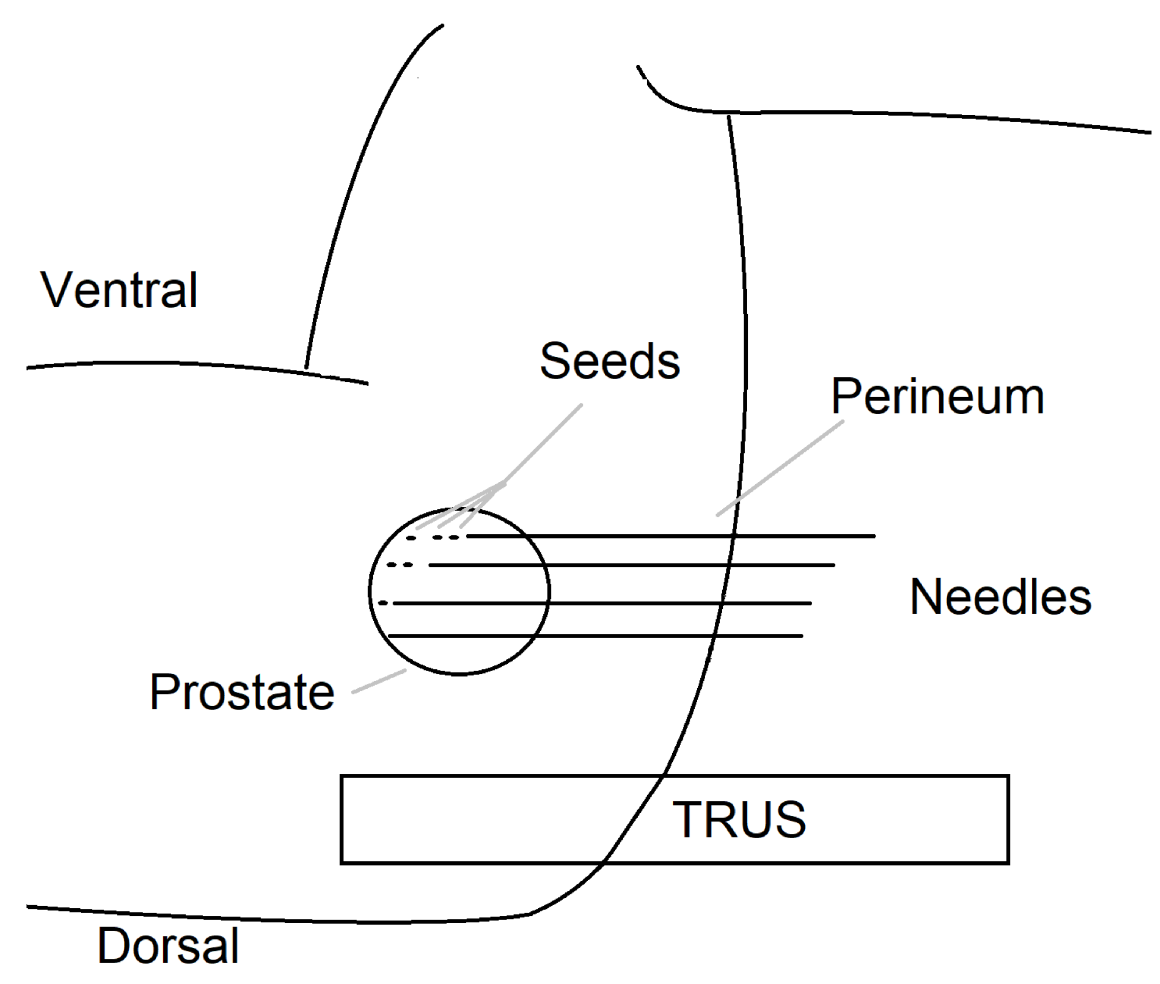

Figure 1.1: Schematic of transperineal permanent implant prostate brachytherapy with transrectal ultrasound guidance in the lithotomy position.

\subsection{Permanent implant prostate brachytherapy}

In a permanent implant prostate brachytherapy (PIPB) procedure, hollow needles are inserted through the perineum with guidance from a trans-rectal ultrasound (TRUS) probe, and treatment seeds are deposited according to a treatment plan (figure 1.1). The number of seeds required for the plan will vary with prostate size and the activity of the seeds.

The treatment plan can be developed in the days or weeks before the procedure (pre-planning), or it can be developed intraoperatively. In the case of pre-planning, the procedure begins with a volumetric image taken of the pelvic region (TRUS is standard but $\mathrm{CT}$ or MRI are also used ${ }^{19}$ ). The target region and organs at risk (OAR) are contoured and treatment planning is performed to determine the placement of treatment seeds that delivers the optimal dose distribution. This optimization can 
be performed manually based on experience and intuition, or it can be automated.

In the case of intraoperative planning, the pelvic region is imaged with the TRUS that will be used for guiding needle placement and the treatment plan is created in real time in the operating room. Both pre-planning and intraoperative planning have been shown to produce similar treatment outcomes,${ }^{20}$ but intraoperative planning allows for the plan to be adapted to changes in target volume and localization as well as the actual placement of each implanted seed which is not always exactly as planned. ${ }^{21}$ As a result, intraoperative planning can achieve greater dose conformity. ${ }^{22}$ An advantage of pre-planning is the ability for the clinic to order the necessary quantity of treatment seeds while the intraoperative technique requires an abundant supply of seeds to be available, which could be impractical for a clinic that performs PIPB infrequently. A post-implant image is taken to verify that the seeds were placed according to plan, and a post-implant dose calculation can be performed. To evaluate the implant, the American Association of Physicists in Medecine (AAPM) and the American Brachytherapy Society (ABS) recommend CT imaging for good source definition. ${ }^{19}$ The optimal timing of the post-implant imaging is dependent on the radionuclide half-life; for ${ }^{125} \mathrm{I}, 42$ days post-implant is recommended. ${ }^{19}$

\subsection{Evaluation of dose}

Absorbed dose is defined as the energy from ionizing radiation that is absorbed per unit mass (see equation (1.1)). Kerma, $K$, is a quantity that is useful in brachytherapy dose calculations and is defined as the expectation value of the energy that is imparted to charged particles by uncharged particles per unit mass in the limit of a small mass such that

$$
K=\frac{\left\langle d E_{t r}\right\rangle}{d m}=\Psi \frac{\mu_{t r}}{\rho}
$$




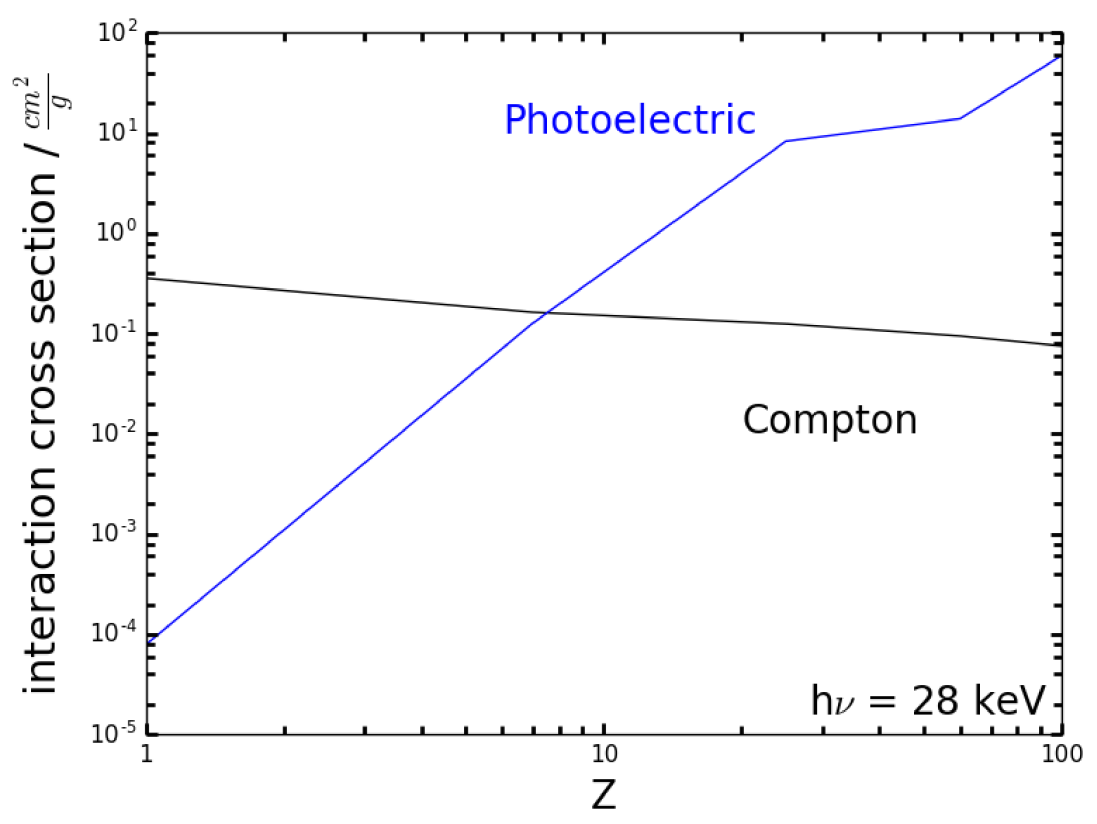

Figure 1.2: The photon cross sections for photoelectric effect and Compton scattering as a function of the atomic number, Z, of the medium for photons with energy $h \nu=$ $28 \mathrm{keV}$ (mean emission energy for ${ }^{125} \mathrm{I}$ ). ${ }^{23}$ From NIST XCOM database. ${ }^{24}$

where $\left\langle E_{t r}\right\rangle$ is the mean energy transferred to charged particles and $m$ is the mass, $\Psi$ represents the energy fluence of the incident particles, and the mass energy transfer coefficient, $\frac{\mu_{t r}}{\rho}$, characterizes the energy that is given as kinetic energy to charged particles by uncharged particles in a medium of density $\rho$.

Energy can be transferred to charged particles in the medium through various physical processes such as Compton scattering and the photoelectric effect, as well as pair and triplet production, but the latter two processes are only relevant for energies much higher than encountered in PIPB. The frequency of the former two processes will depend on the characteristics of both the incident particles and the medium (shown in figure 1.2).

The difference between kerma and dose lies in the distinction between the energy transferred, $E_{t r}$, and the energy absorbed, $E_{a b} . E_{t r}$, and therefore kerma, includes all energy that is imparted to charged particles in the mass, regardless of the eventual 
endpoint of the energy, whereas $E_{a b}$, and therefore dose, includes only that energy which is eventually imparted to the mass by charged particle interactions. The difference is attributable to energy that is transferred to the mass but then carried away.

In brachytherapy dose calculations it is often assumed that energy transferred to a charged particle is deposited to the mass locally, in which case the approximation can be made that

$$
\begin{gathered}
E_{a b} \approx E_{t r} \\
D \approx K=\Psi \frac{\mu_{t r}}{\rho} .
\end{gathered}
$$

While the mass energy transfer coefficient in equation (1.2) accounts for all energy that is imparted as kinetic energy to a charged particle, the mass energy absorption coefficient, $\frac{\mu_{e n}}{\rho}$, characterizes only the energy given as kinetic energy to the charged particles that is ultimately deposited locally and not energy that escapes through radiative processes. The two quantities are related as follows

$$
\frac{\mu_{e n}}{\rho}=\frac{\mu_{t r}}{\rho}(1-\bar{g})
$$

where $\bar{g}$ is the mean radiation fraction, the fraction of energy given to charged particles that is subsequently radiated in the form of photons, averaged over the spectrum of energies of charged particles set in motion by primary photons. ${ }^{25}$ Similarly, while kerma accounts for all energy transferred to a medium, the collision kerma $K_{\text {col }}$ does not include radiative losses such that

$$
\begin{aligned}
K_{c o l} & =K(1-\bar{g}) \\
& =\Psi \frac{\mu_{t r}}{\rho}(1-\bar{g}) \\
K_{c o l} & =\Psi \frac{\mu_{e n}}{\rho}
\end{aligned}
$$


It follows from equation (1.3) and equation (1.5) that if electrons are assumed to deposit energy locally and $\bar{g}<<1$ the approximation can be made that

$$
D \approx K \approx K_{c o l}=\Psi \frac{\mu_{e n}}{\rho} .
$$

The value of $\bar{g}$ is not commonly tabulated, but the above inequality $(\bar{g}<<1)$ can be shown to hold true through a related quantity, the radiation yield. The radiation

yield, $Y\left(E_{K}\right)$, is the fraction of the initial kinetic energy $\left(E_{K}\right)$ of a charged particle that is emitted as radiation so that $\bar{g}$ is the mean value of $Y\left(E_{K}\right)$ for the spectrum of charged particle kinetic energies. If it is shown that $Y\left(E_{K}\right)<<1$ for the energy spectrum relevant for brachytherapy then $\bar{g}$, the spectrum average of $Y\left(E_{K}\right)$, must also necessarily be much less than 1 .

The validity of the assumptions inherent to equation (1.6) for brachytherapy is addressed in figure 1.3, which shows that for energies typical in brachytherapy the radiation yield is much less than 1 and the range of an electron is less than $1 \mathrm{~mm}$ which is the low end of the length-scale that the clinical dose calculation is performed on, supporting the use of the approximations in equation (1.6).

\subsubsection{TG-43 formalism}

In 1995 the AAPM Radiation Therapy Committee Task Group 43 released a set of recommendations for the dose calculations using interstitial brachytherapy sources, defining both a standard data set for parameters and an established protocol for dose calculations commonly referred to as the TG-43 formalism or simply 'TG- 43 '. ${ }^{27}$ The TG-43 formalism calculates a dose distribution around a single treatment seed in a volume of homogeneous water large enough to account for scatter. This dose distribution is superimposed at each seed location to give a total dose to the target area. This formalism, with a few updates ${ }^{28-30}$ is the current clinical standard for 


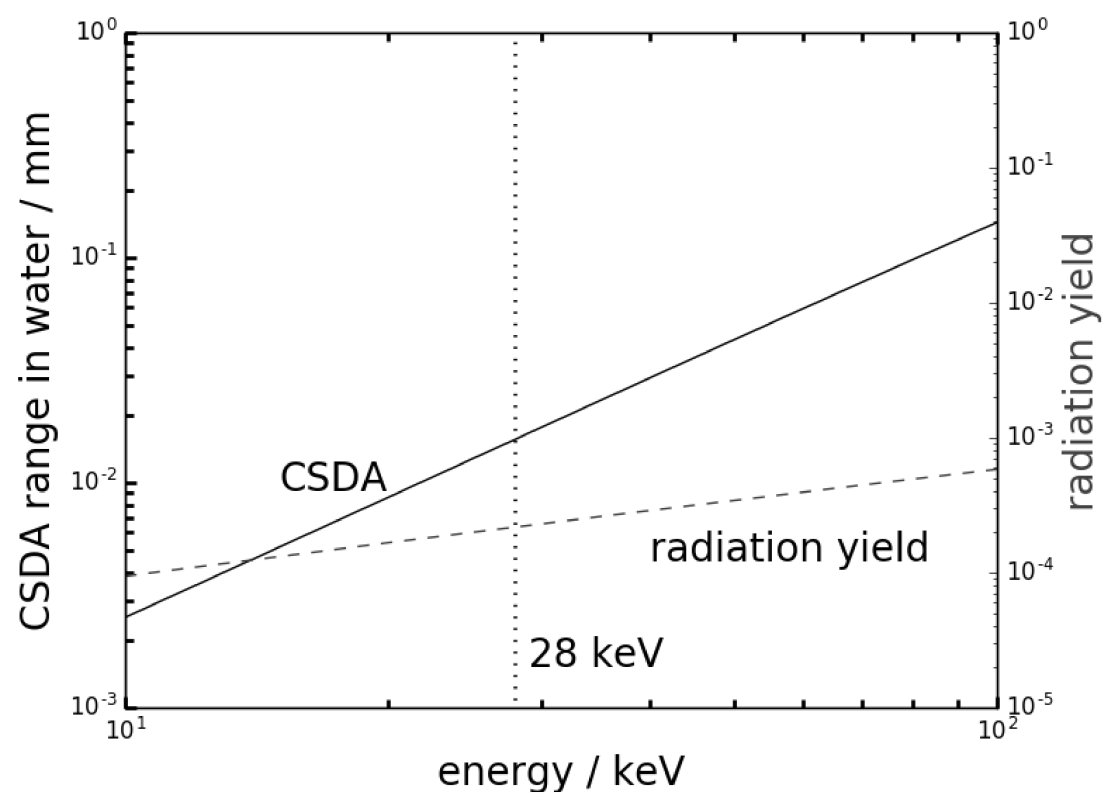

Figure 1.3: Continuous slowing down approximation range in water (solid, left) and radiation yield (dashed, right) of an electron as a function of kinetic energy. The mean emission energy of ${ }^{125} \mathrm{I}$ is represented by the vertical dotted line. The range of an electron with energy in the brachytherapy range is less than the length scale of interest for clinical dose calculations. Data from NIST ESTAR. ${ }^{26}$ 
interstitial brachytherapy dose calculations.

\subsubsection{Dose metrics}

To evaluate a treatment plan it is often useful to quantitatively describe the dose delivered to a structure rather than to a point. Some metrics characterize the dose to a contiguous volume such as a $1 \mathrm{~cm}$ segment of the rectum wall, but many of the common metrics are best represented with a dose-volume histogram (DVH).

A generic dose-volume histogram is presented in figure 1.4. A DVH shows on the $y$-axis the percentage of the target volume that receives a dose of at least $x$, which is a convenient method of reducing a three dimensional distribution of the dose to all voxels in a volume down to two dimensions. A drawback of the DVH representation is that any spatial information of individual voxels is lost, but broad volumetric trends are easily visualized and described by various metrics. Two major groups of DVH-based metrics exist: the $\mathrm{D}_{x}$ and the $\mathrm{V}_{x}$ metrics. The metric $\mathrm{D}_{x}$ describes the minimum dose received by the hottest $x$ percent of the volume, $\mathrm{V}_{y}$ describes the percent volume receiving a dose of at least $y$. The value of $y$ may represent either an absolute dose, or a percentage of the prescription dose. The latter convention is adopted for the remainder of this work. The value of DVH-based metrics is two-fold; they conveniently quantify complex 3D dose distributions with a single number, and some metrics have been shown to be predictors of patient outcomes. ${ }^{31}$

\subsubsection{Implant criteria}

The most common quantities for evaluating an implant are $D_{90}$ and $V_{100}$ (see figure 1.4). $D_{90}$ has been shown to be an indicator of long-term outcomes in PIPB. It was demonstrated that there exists a correlation between $\mathrm{D}_{90}$ value and freedom from biochemical failure. ${ }^{31} \mathrm{~A} \mathrm{D}_{90}$ value below the prescription dose was found to reduce the rate of freedom from biochemical failure by $15 \%{ }^{32,33}$ and $\mathrm{D}_{90}$ was found to be 


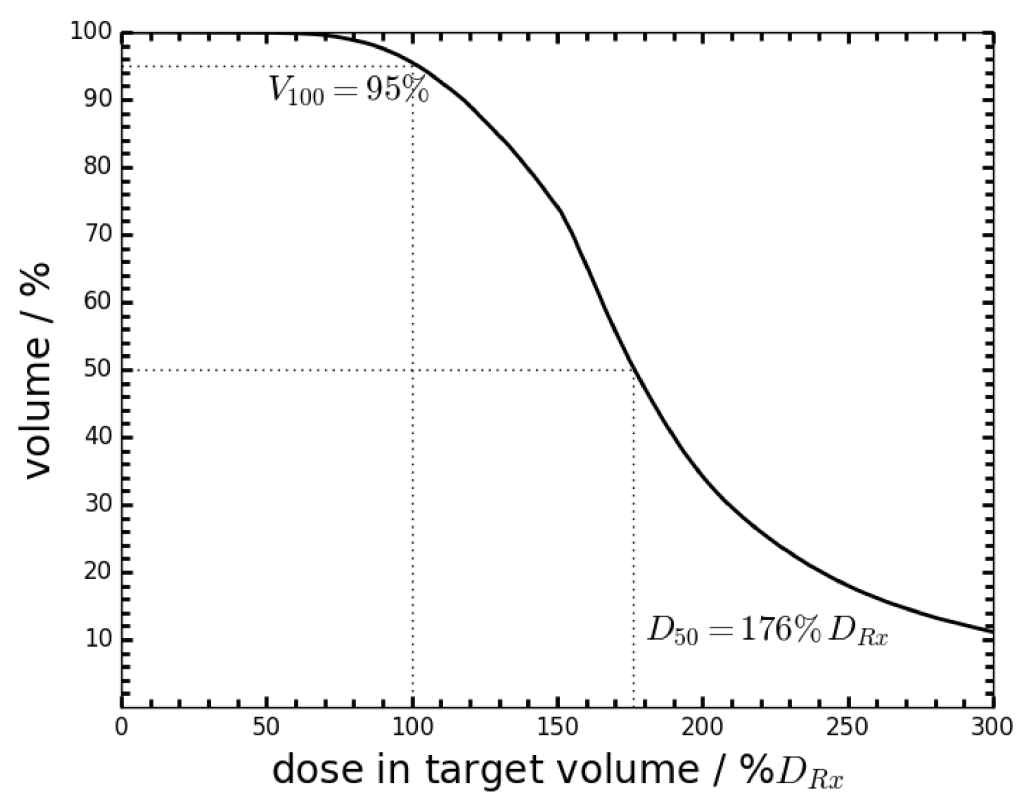

Figure 1.4: A generic DVH illustrating the concepts of $\mathrm{D}_{x}$ and $\mathrm{V}_{x}$ metrics. E.g. $\mathrm{D}_{50}$ represents the minimum dose received by the hottest $50 \%$ of the target volume, $\mathrm{V}_{100}$ represents the $\%$ of the target volume receiving a dose $\geq D_{\mathrm{Rx}}$ (the prescribed dose).

the most significant predictor of freedom from biochemical recurrence at 10 years. ${ }^{34}$ In addition to good coverage of the prostate, a good implant will also spare any organs at risk (OAR) from receiving unnecessary dose. Recommendations for what constitutes a good PIPB implant have been made by the AAPM ${ }^{19}$ and the American Brachytherapy Society (ABS) ${ }^{35}$ and are summarized in table 1.2.

\subsubsection{Challenges in brachytherapy dose calculations}

The standard for dose evaluation of PIPB was last updated in $2017,{ }^{30}$ but is still primarily based on the original TG- 43 recommendations from $1995 .{ }^{27}$ While the TG-43 formalism is successful at estimating dose in clinically useful times, the approximation of a static and infinite homogenous water medium is known to result in inaccuracies, particularly where the volume is changing or where there is a medium that is dissimilar to water. 
Table 1.2: Summary of implant criteria.

\begin{tabular}{ll}
\hline Criterion & Recommendation \\
\hline \hline Prescription Dose, $\mathrm{D}_{R x}$ & ${ }^{125} \mathrm{I}: 145 \mathrm{~Gy}^{\dagger}, 140-160 \mathrm{~Gy}{ }^{\ddagger}$ \\
& ${ }^{103} \mathrm{Pd}: 125 \mathrm{~Gy}^{\dagger}, 110-125 \mathrm{~Gy}{ }^{\ddagger}$ \\
& ${ }^{131} \mathrm{Cs}: 100-125 \mathrm{~Gy}^{\dagger}$ \\
& $\mathrm{V}_{100}>95 \%^{\dagger}$ \\
& $\mathrm{D}_{90}>\mathrm{D}_{\mathrm{Rx}}^{\dagger}$ \\
& $V_{150} \leq 50 \%^{\dagger}$ \\
& $\mathrm{D}_{2 \mathrm{cc}}<\mathrm{D}_{\mathrm{Rx}^{\dagger}}$ \\
Rectum & $\mathrm{D}_{0.1 \mathrm{cc}}<150 \% \mathrm{D}_{\mathrm{Rx}}^{\dagger}$ \\
& $\mathrm{V}_{100}<1 \mathrm{cc}^{\ddagger}$ \\
& $\mathrm{D}_{10}<150 \% \mathrm{D}_{\mathrm{Rx}}{ }^{\dagger}$ \\
Urethra & $\mathrm{D}_{30}<130 \% \mathrm{D}_{\mathrm{Rx}}^{\dagger}$ \\
& $\mathrm{V}_{150} \operatorname{minimal}^{\ddagger}$ \\
\hline
\end{tabular}

${ }^{\dagger} \mathrm{AAPM}$ recommendations, ${ }^{\ddagger} \mathrm{ABS}$ consensus guidelines $D_{\# c c}$ is the dose to the hottest \# $\mathrm{cm}^{3}$ of tissue

The static volume approximation can introduce inaccuracies in interstitial brachytherapy dose calculations because inflammation resulting from the implant procedure can cause edema, a natural response where extracellular fluid floods a region to aid recovery after trauma, manifesting as localized swelling. The characteristics of this edema response can vary widely between patients and institutions performing PIPB. ${ }^{19}$ In some cases the edema is observed to resolve linearly with time, ${ }^{36,37}$ but most studies suggest edema resolution follows an exponential decay pattern. ${ }^{19,38,39}$

Modelling edema adds complexity to dose calculations. One consideration is the change in prostate volume. The increase in volume or 'edema magnitude' $(\Delta)$ is described in the literature as either the percent increase relative to the reference volume (e.g., 25\%) or as a fraction of the reference volume (e.g., 1.25). The latter convention will be adopted for the remainder of this work so that $\Delta=\frac{V}{V_{r e f}}$. The edema magnitude is time-dependent, but commonly the edema magnitude is described solely by the maximum magnitude, $\Delta_{\max }$. 
The reported average $\Delta_{\max }$ varies considerably between studies ranging from $1.18^{40}$ to $1.58^{38}$ and in extreme individual cases a patient can experience a doubling of prostate volume. ${ }^{39}$ The fluid that is causing the swelling will absorb energy that according to the treatment plan should be deposited in target tissue. Consequently the calculated dose overestimates the delivered $\mathrm{D}_{90}$ by as much as $10 \% .{ }^{38} \mathrm{~A}$ second consideration is that the edema is occurring after the brachytherapy seeds have been implanted, so the deformation of the tissue displaces the seeds from their planned treatment position.

Previous efforts to model anatomical changes due to edema have followed comprehensive imaging studies to monitor edema over the treatment course. ${ }^{38-43}$ Attempts to create predictive models of edema have been limited. One approach transformed a CT image to be representative of an edematic prostate, ${ }^{44}$ but the focus was exclusively on quantifying the physical effects of edema and lacked any dosimetric analysis. Another approach used finite-element modelling to track individual volume elements of the prostate over time as a function of bio-mechanical processes taking place during edema. ${ }^{45}$

The dosimetric effect of edema has been investigated using TG- 43 by calculating an accumulated dose distribution by either introducing a time dependence to the seedto-point distance component ${ }^{43,46-49}$ or by summing DVHs calculated on sequential geometries obtained by multiple imaging, ${ }^{38,50}$ with reductions in $\mathrm{D}_{90}$ of approximately $5 \%$ on average when compared to a clinical (static) TG-43 dose calculation. Edema was found to significantly detract from implant quality in approximately $10 \%$ of all patients. ${ }^{38,51}$ For patients with large and long-lasting edema, the edema is associated with a reduction in $D_{90}$ of as much as a $10 \% .{ }^{38}$

The TG-43 approximation that all media are water can introduce inaccuracies because unique media have different interaction cross sections and will accordingly have different radiation transport properties. Figure 1.2 illustrates that for photon 
energies near the emission energies of ${ }^{125} \mathrm{I}$, the total photon cross section has a strong dependence on the atomic number, Z, of the medium because the photoelectric effect is a major contributor to the cross section at these energies. The interaction cross section is inversely related to the mean free path, so it follows that if the cross section has a strong dependence on $\mathrm{Z}$, so will the transport of radiation. Consequently the mean free path of a photon in water is greater than it would be in a high-Z material so the TG-43 approximation of using water for all media will underestimate the local deposition of energy and overestimate the mean free path of the photon. Two instances of high-Z materials encountered in PIPB are treatment seeds ${ }^{52-54}$ and calcium deposits. ${ }^{53}$

Many soft tissues have elemental compositions very similar to water and the approximation of the medium as water introduces only a small discrepancy, but for higher-Z materials the approximation is poor. The source capsules can have very high $\mathrm{Z}$-values $\left(\mathrm{Z}=22\right.$ for a titanium capsule) compared to water $\left(\mathrm{Z}_{\text {eff }} \approx 7.4\right)^{55}$ and correspondingly do not transport radiation in the same way. The attenuation of radiation by a seed other than the originating source (interseed attenuation; ISA) cannot be accounted for by the TG- 43 formalism approach of superimposing waterbased dose distributions. Failing to account for this extra attenuation can result in overestimation of $D_{90}$ by as much as $5 \% .53,54$

Figure 1.5 shows that the mass energy absorption coefficient, $\frac{\mu_{e n}}{\rho}$, which characterizes the amount of energy a medium absorbs, for water is comparable to that of prostate and soft tissues but poorly represents media such as calcification or bone.

In a large cohort study it was found that roughly $11 \%$ of PIPB patients had a non-negligible volume of intraprostatic calcification (IC). ${ }^{56}$ IC has been observed to reduce $\mathrm{D}_{90},{ }^{57,58}$ which is linked to worse treatment outcomes (see section 1.4.3). The relatively high- $Z$ calcium is more opaque to radiation than normal prostate tissue resulting in a dose shadowing effect behind the calcification. Figure 1.5 shows the 


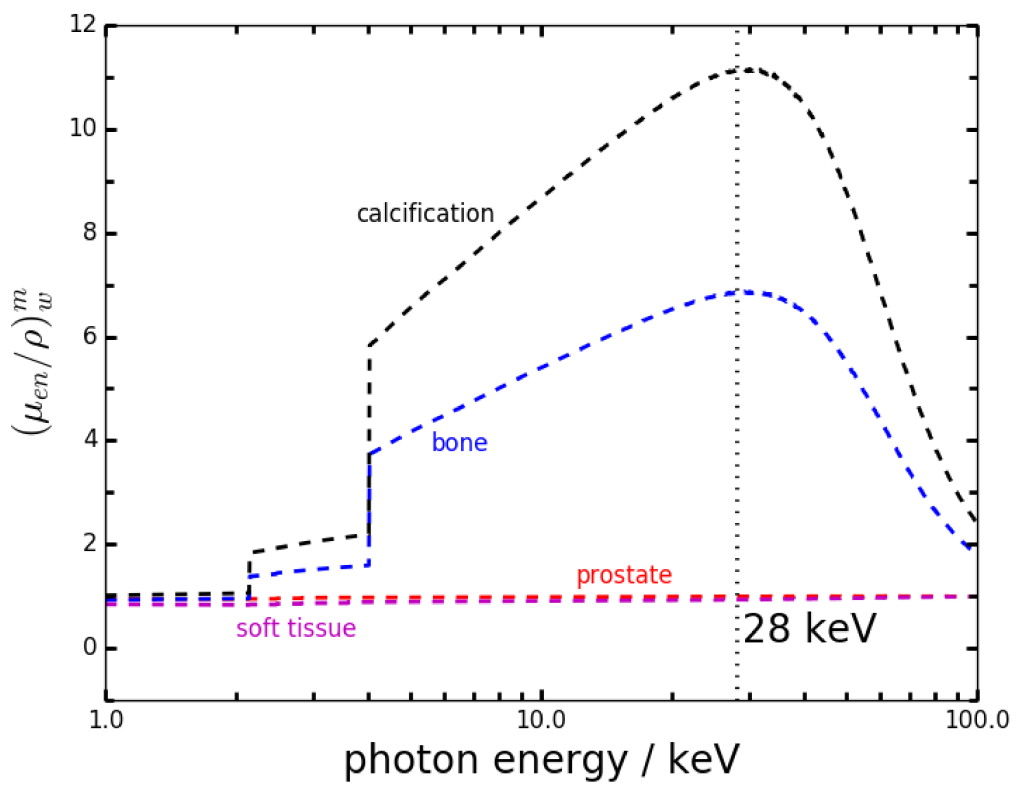

Figure 1.5: The mass-energy absorption coefficient $\left(\mu_{e n} / \rho\right)$ characterizes the amount of energy absorbed by a medium as radiation passes through it. Shown here are the $\mu_{e n} / \rho$ values for different media divided by the $\mu_{e n} / \rho$ value for water, calculated with EGSnrc application $g$. The dashed vertical line represents the mean ${ }^{125}$ I photon energy. Source of data is egs_brachy distribution. ${ }^{59}$

mass-energy absorption coefficient for IC is an order of magnitude larger than that of water for photon energies near the mean photon emission energy of ${ }^{125} \mathrm{I}$.

\subsubsection{Model-based dose calculation algorithms}

To address the shortcomings of the TG-43 formalism several approaches have been developed that use detailed models of the patient tissue composition to perform dose calculations. These model-based dose calculation algorithms (MBDCAs) use a variety of techniques ${ }^{60}$ to model the radiation transport including deterministic solving of a discretized Boltzmann linear transport equation, ${ }^{61}$ a collapsed cone convolution superposition approach, ${ }^{62}$ and Monte Carlo (MC) simulations. ${ }^{56,63}$

MC dose calculation is a model-based technique that simulates radiation transport as a series of random events by successively sampling probability distributions. MC 


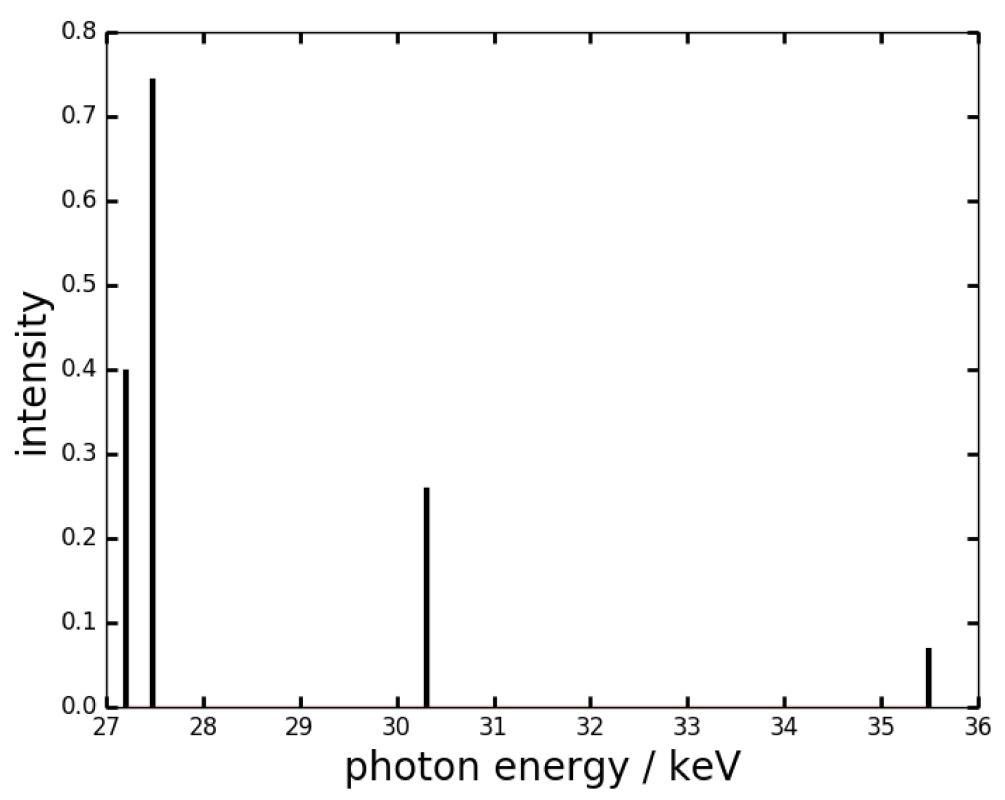

Figure 1.6: NCRP ${ }^{125}$ I photon spectrum from NCRP Report No. 58 [Ref. 65]

simulation has previously been applied to investigations of PIPB. ${ }^{45,56,64}$ In the context of brachytherapy these probability distributions can describe many phenomena. Consider figure 1.6, which shows the spectrum of the energies of photons emitted from an ${ }^{125}$ I source which represents a probability distribution for the energy of a newly created photon.

The likelihood of the newly created photon passing through the seed capsule and into the patient's tissue is a function of both the photon's energy and the material properties of the capsule, so the probability is determined by both particle and modelbased attributes. The relative cross sections give the probabilities of which interaction will occur when the photon does interact and a higher total cross section corresponds to a photon that will be more likely to interact and therefore have a shorter mean free path.

After an interaction, any resulting particles (e.g., a scattered photon) that are created in the simulation are tracked until they interact, exit the geometry, or have low enough energy that it can be assumed the energy is deposited locally. A photon gen- 
erally terminates via the photoelectric effect after only a few interactions, compared to an electron which generally loses only a small amount of energy per interaction and can interact many times. Consequently, photon interactions are simulated as discrete events, but it is common for MC codes to simulate electron transport by 'condensing' many interactions into a single event to increase efficiency. ${ }^{66}$ The complete chain of interactions stemming from a single starting particle constitutes a history. The number of histories composing a MC simulation is defined by the user. Depending on the motivation, millions or billions of histories can be used. Monte Carlo simulations have the potential to be highly accurate because they include a realistic representation of the patient geometry while faithfully simulating the underlying physics that contribute to dose deposition, but this comes at a cost of increased computational time compared to TG-43.

To speed up calculations, many MC codes use a technique called track-length scoring $^{67}$ to evaluate the dose in a voxel. For brachytherapy energies (and the associated short electron ranges and low radiative loss fractions) equation (1.6) is valid ( $D \approx \Psi \frac{\mu_{e n}}{\rho}$ ). The energy fluence $\Psi$ is $E \cdot \phi$ where $\mathrm{E}$ is the particle energy and $\phi$ is the particle fluence in the volume. The average particle fluence in a volume can be expressed as

$$
\phi=\frac{\sum_{i} t_{i}}{V}
$$

where $V$ is the volume and $t_{i}$ is the length of track $i$ through the volume, which can be calculated quickly in a MC simulation. Estimating dose in this manner can increase simulation efficiency by a factor of up to 20 compared to scoring individual interactions. ${ }^{67}$ 


\subsection{Thesis purpose}

Research in brachytherapy dose calculations using MBDCAs has shown that TG-43 dose calculations, the current clinical standard, can significantly overestimate dose by not accounting for interseed attenuation ${ }^{52-54}$ and tissue composition. ${ }^{54,56,58}$ For PIPB patients the TG- 43 approach overestimates $\mathrm{D}_{90}$ by around $5 \%$ on average, ${ }^{54,56}$ and by much more in patients with significant volumes of calcification where $\mathrm{D}_{90}$ was found to be reduced by up to $37 \%^{58}$

Edema has also been linked to reduced $\mathrm{D}_{90}$ when compared to a static dose calculation based on images taken 30 days post-implant. It is expected that the magnitude of the dosimetric effect depends on the characteristics of the edema, which vary considerably in the literature. ${ }^{37-42,68-70}$

While the effects of tissue composition and edema have been studied separately, investigation of both effects together by performing dose accumulation using MBDCA techniques has been limited. Mountris et al. ${ }^{45}$ developed models of 15 patients and using finite element techniques explored characteristics of edema resolution as a function of the physical properties (Young's modulus, Poisson's ratio) of the prostate tissue. They proposed that inter-patient variation in these physical properties influences the edema resolution pattern. For a small Young's modulus the resolution appears linear as observed in some studies ${ }^{36,37}$ while a larger Young's modulus is associated with the exponential resolution observed by others. ${ }^{19,38,39}$ Mountris et al. ${ }^{45}$ showed their model was able to match a previously published ${ }^{41}$ post-implant time series of volumes. By re-sampling the finite element mesh at various stages of edema, Virtual Patient Models (VPMs) were generated to be used for MC dose calculations and these individual dose distributions were combined into a 'dynamic' dose representing the dose deposited in the changing target volume. Unfortunately, the method of combining the doses into a dynamic dose is not described. The dose accumulation process is not trivial because the individual doses should be appropriately weighted 
and mapped to a single reference. The method of mapping can introduce errors, with energy mapping ${ }^{71}$ being superior to direct dose mapping, particularly in regions with high gradients of density or dose. ${ }^{72}$

This thesis complements the work of Mountris et al. with more patients and edema models (120 patients, and 10 models of edema from the literature), an accurate energy mapping method, ${ }^{73}$ and an alternate approach to generating VPMs. Towards this end, this thesis presents a complete framework for calculating accumulated doses in the presence of edema based on a post-implant CT image using the brachytherapyspecific MC code egs_brachy. ${ }^{59}$ egs_brachy is a universally-accessible code that is free and open source. The goal of this work is to demonstrate the framework for evaluating dose distributions delivered to a changing geometry during brachytherapy by applying the framework to an investigation of the dosimetric effects of calcification during PIPB treatment.

\subsection{Thesis outline}

Chapter 1 provided an introduction to brachytherapy and dose evaluation. PIPB and current dosimetry practices were discussed and an introduction to model-based dose calculation was given. Chapter 2 describes the methods used to calculate a dynamic dose accounting for edema in more detail including the techniques to extrapolate deformed patient models, calculate dose distributions in a fixed model using MC simulation, dose mapping and the accumulation of dose. Chapter 3 presents the results of dynamic dose calculations in the presence of edema for several models of edema. The impact of the presence of calcifications in the prostate is also investigated. Chapter 4 contextualizes the results within contemporary literature and evaluates the strengths and limitations of the presented framework and also summarizes the major findings of the thesis and proposes opportunities for advancing 
this research further. 


\section{Chapter 2}

\section{Methods}

An overview of the process for calculating an accumulated dose in the presence of edema is shown in figure 2.1. This section will explain the details of this process.

\subsection{Patient data}

A cohort of $613{ }^{125}$ I PIPB patients who were treated at Centre Hospitalier Universitaire de Québec from 2003 to 2012 were previously analyzed. ${ }^{56,74}$ For each patient there was a CT image that was acquired 30 days post-implant and a treatment plan containing air kerma strength, seed locations, and contours of the prostate, rectum, bladder and bones. Prostate volume ranged from 12.7 to $84.8 \mathrm{~cm}^{3}$ with a median of $36 \mathrm{~cm}^{3}$. The number of implanted seeds ranged from 23 to 88 with a median of 53 . Of the cohort, 60 patients were found to have prostates with $>0.3 \%$ by volume intraprostatic calcification. These patients comprise the IC (intraprostatic calcification) sub-cohort. An additional 60 patients were selected randomly from the remainder of the cohort as the NC (no calcification) sub-cohort for comparison.

In previous work by Miksys et al.,${ }^{74}$ patient CT images were converted into voxelized virtual patient models (VPMs), each voxel having an associated medium and density. The resolution of the voxels in the VPM was chosen to match the resolution of the image (ranging from 0.31 to $0.38 \mathrm{~mm}$ in the transverse plane and from 2 to $2.5 \mathrm{~mm}$ on the superior-inferior axis). Using a combination of the contours and CT number (post-processed to mitigate metallic artifacts from treatment seeds ${ }^{74}$ ) appro- 


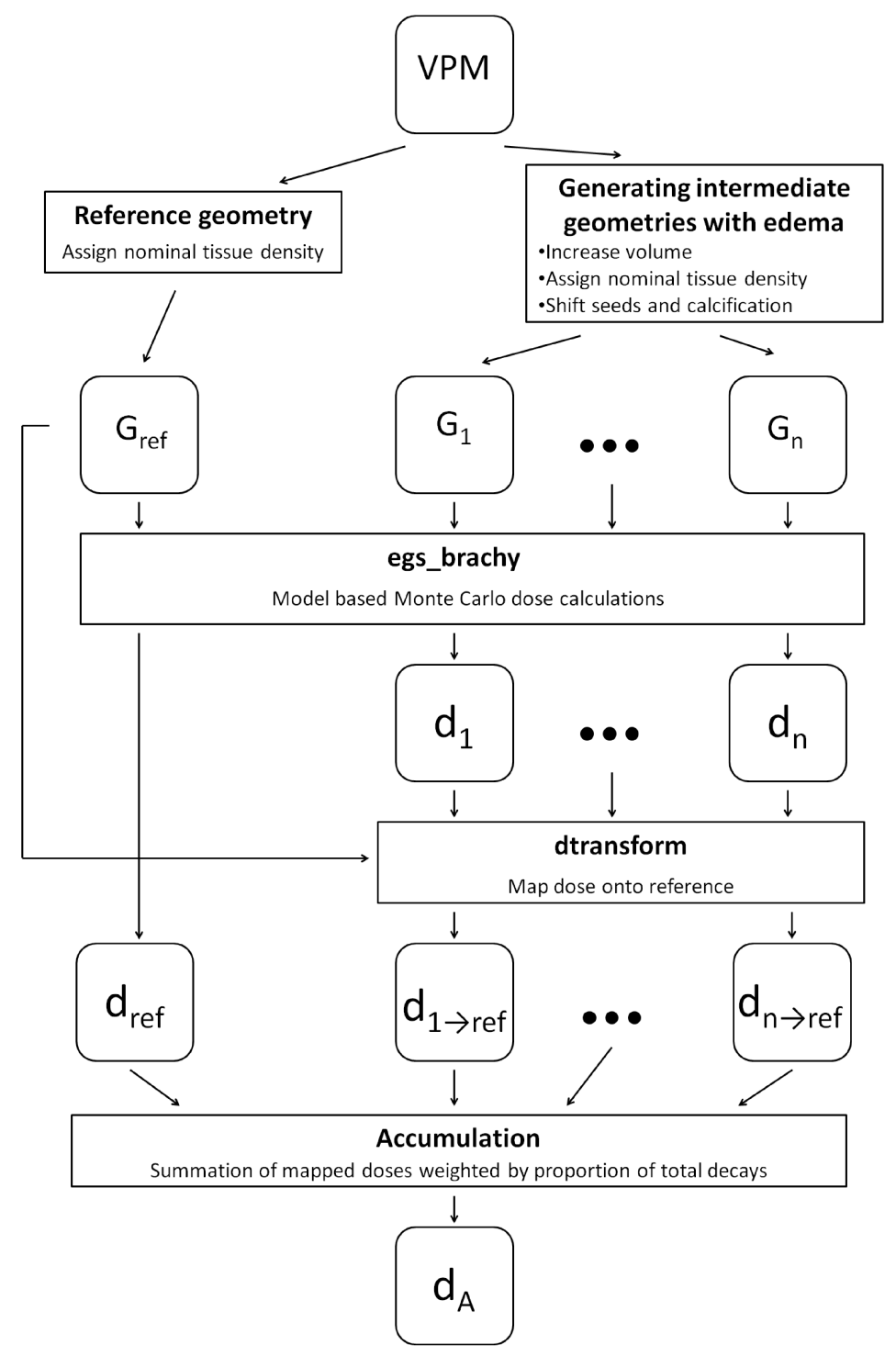

Figure 2.1: Overview of the dynamic dose calculation process. A CT-based virtual patient model is used to generate intermediate geometries, $G$. The MC code egs_brachy ${ }^{59}$ is used to calculate a dose distribution, $d$, for each geometry. Calculated dose distributions are mapped to common reference geometry, $G_{r e f}$, using the dose mapping code dtransform ${ }^{73}$ resulting in mapped dose distributions $d_{n \rightarrow r e f}$. The mapped dose distributions are combined into an accumulated dose distribution, $d_{r e f}^{a c c}$. 
Table 2.1: Tissue assignment scheme for generating full-tissue VPM from pelvic CT. ${ }^{56}$

\begin{tabular}{lll}
\hline Region & Tissue & Mass density range \\
\hline \hline Target & Prostate & $\leq 1.14 \mathrm{~g} / \mathrm{cm}^{3}$ \\
& 50\% Prostate $/ 50 \%$ Calcification & $1.14-1.27 \mathrm{~g} / \mathrm{cm}^{3}$ \\
& Calcification & $\geq 1.27 \mathrm{~g} / \mathrm{cm}^{3}$ \\
Urethra & Prostate & All \\
Rectum & Rectum & All \\
Bladder & Urinary bladder(empty) & All \\
Remainder & Mean male soft tissue & $\leq 1.14 \mathrm{~g} / \mathrm{cm}^{3}$ \\
& Cortical Bone & $\geq 1.14 \mathrm{~g} / \mathrm{cm}^{3}$ \\
\hline
\end{tabular}

priate tissues and densities are assigned to each voxel as shown in table $2.1{ }^{56}$ There is also an artificial medium, $50 \%$ prostate $/ 50 \%$ calcification that is a homogenous blend of equal parts prostate and calcification tissues to represent voxels partially filled with calcification or with sub-voxel sized deposits of micro-calcification. These VPMs are the starting point for the work done in this thesis.

\section{2 egs_brachy}

The MC code egs_brachy ${ }^{59}$ is a fast EGSnrc-based application which performs advanced model based dose calculations by simulating transport of radiation from brachytherapy sources. As a brachytherapy-specific code, egs_brachy includes many features useful for simulating brachytherapy treatments, such as particle recycling ${ }^{75}$ and voxel volume corrections in the region of treatment seeds. ${ }^{59}$ egs_brachy has previously been validated in comparisons against MC codes BrachyDose, ${ }^{59}$ PENELOPE $^{76}$ and MCNP5. ${ }^{77}$

A photon initialized in the active source of a seed might never leave the seed to contribute to dose in a scored region. To increase simulation efficiency, a photon that does escape a seed is 'recycled' to every other seed as if it were also created in those 
seeds as well. This particle recycling technique has been used in brachytherapy to increase the proportion of simulation time that is spent transporting photons that will contribute to scored quantities, thereby increasing the efficiency by as much as $60 \%$ for ${ }^{125}$ I sources. $^{75}$

A complication to dose calculations in brachytherapy is that the proximity of the treatment sources to the target volume means that there are voxels partially filled by both medium, where dose should be scored, and the source, where energy is not absorbed by the target. Voxel volume correction is a technique that is built into egs_brachy which corrects for this by randomly sampling points in the voxel to determine the fraction of volume that is taken up by a source. ${ }^{59}$ Voxel volume correction was not performed to keep consistency with the dose mapping which does not handle partially filled voxels. The loss of some accuracy in these voxels is not clinically important in PIPB dose calculations because the proximity to a source means the dose will be extremely high.

Photon cross sections are taken from the NIST XCOM database, ${ }^{24}$ and massenergy absorption coefficients are calculated with the EGSnrc application $g$. The NCRP ${ }^{125}$ I photon spectrum ${ }^{65}$ distributed with egs_brachy was used to initialize photons in treatment seeds rather than the TG- 43 spectrum, ${ }^{28}$ as it leads to better agreement with measurements. ${ }^{78}$ The seed model was chosen according to what treatment seed was implanted clinically. The SelectSeed 130.002 [Nucletron, the Netherlands] and the ProstaSeed 125SL [Mills Bio. Pharm, USA] seeds were used in 101 and 19 of the 120 treatments, respectively.

The parameters used for MC simulations with egs_brachy are summarized in Table 2.2. For each patient the edema time-course was discretized into intermediate geometries and the doses to the intermediate geometries were calculated on the Carleton University Physics Research Compute Cluster using egs_brachy with $10^{7}$ effective $a b$ initio (starting particle initiated in the source) histories per seed yielding average 
Table 2.2: Monte Carlo methods in accordance with TG-268 guidelines. ${ }^{79}$

\begin{tabular}{lll}
\hline Checklist item & Item name & Descriptions \\
\hline 2,3 & Code & egs_brachy, ${ }^{59}$ v2017.09.15 \\
\hline 5 & Timing & 5 hours for one CPU $($ Dell 1950) \\
\hline 8 & Source description & NCRP ${ }^{125}$ I spectrum initialized photons in: \\
& & Mills Bio. Pharm., ProstaSeed, 125SL ${ }^{80}$ \\
& Nucletron, SelectSeed, 130.002 $\left.{ }^{80}\right)$
\end{tabular}


dose uncertainties of $<2 \%$ in approximately 5 hours.

\subsubsection{Outputs}

The MC code egs_brachy normalizes the scored dose in a voxel, $j$, by the number of histories to determine a dose per history to that voxel, $D^{j}$, which is the output of the simulation. The dose per history can be converted to an absolute dose $D_{\text {tot }}^{j}$ for the entire treatment using a dose scaling factor

$$
D_{\text {tot }}^{j}=D^{j} \frac{\left[S_{k}\right]_{\max }}{S_{k}^{\text {hist }}} \tau .
$$

For a permanent implant, the dose scaling factor is a function of the radionuclide

mean lifetime, $\tau$, the air kerma strength of the sources at implant time, $\left[S_{k}\right]_{\max }$, and the air kerma strength per history, $S_{k}^{\text {hist }}$ which is characteristic of the source model and can be estimated with Monte Carlo methods. ${ }^{83}$

Similarly, the uncertainties are calculated on a history-by-history basis.

$$
s_{\bar{X}}=\sqrt{\frac{1}{N-1}\left(\frac{\sum_{i=1}^{N} X_{i}^{2}}{N}-\left(\frac{\sum_{i=1}^{N} X_{i}}{N}\right)^{2}\right)},
$$

where $X_{i}$ is a scored quantity in a voxel and $\mathrm{N}$ is the number of histories. Increasing the number of histories decreases the uncertainty, but it also increases the computational time required for a simulation.

\subsubsection{Comparison of egs_brachy to BrachyDose}

Dose distributions calculated with egs_brachy on patient geometries from this study

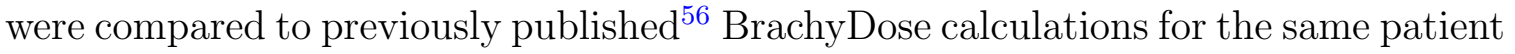
database (figure 2.2). The comparison is made in terms of $\triangle D_{L O C A L}$ as described by 


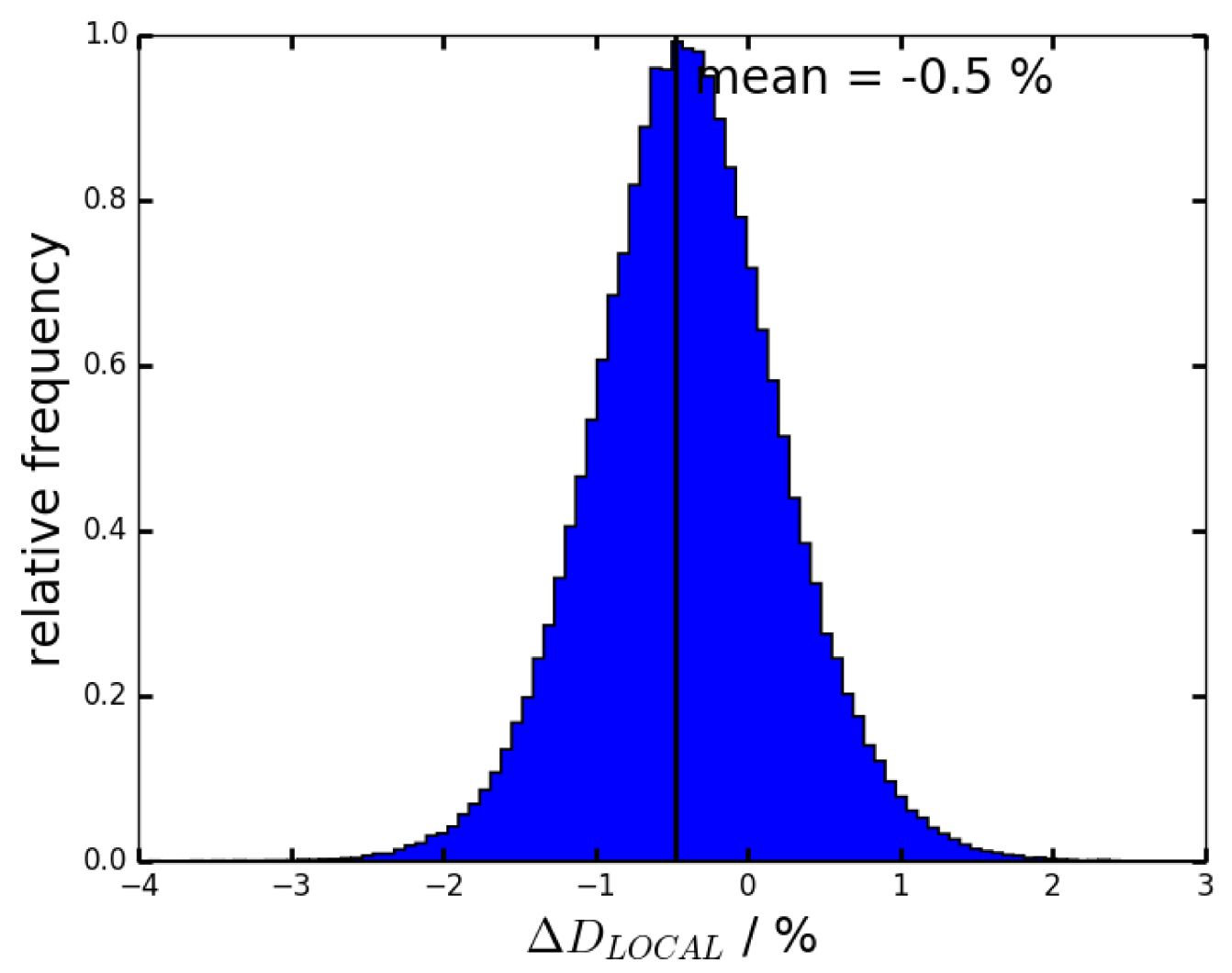

Figure 2.2: Voxel dose differences between egs_brachy and BrachyDose calculations for an example patient treatment from the dataset, presented as $\triangle D_{L O C A L}$ where the BrachyDose calculated distribution is in the denominator. The $0.5 \%$ systematic offset between the two codes agrees with previously published results ${ }^{59,77}$ and is an expected level of discrepancy between these two different $\mathrm{MC}$ dose calculation codes.

Ma et al. ${ }^{76}$ where

$$
\Delta D_{L O C A L}=\frac{D_{2}(r)-D_{1}(r)}{D_{1}(r)},
$$

with $D_{n}(r)$ being the dose calculated in the individual voxel located at position $r$ using either of two codes. Evaluating the distribution of $\triangle D_{L O C A L}$ for all of the voxels in a target(figure 2.2), good agreement is found with a systematic difference of about $0.5 \%$ as seen in previous comparisons between these two codes. ${ }^{59,77}$ 


\subsubsection{Simulating TG-43 in egs_brachy}

egs_brachy can mimic the conditions of a TG-43 dose calculation by simulating treatment to an all-water VPM with one seed at a time. In this way the dose to water from a single seed is evaluated and summed with contributions from other seeds, without any inter-seed effects. In theory, this approximation should give the same result as the analytic TG-43 solution. In practice, small discrepancies in calculated dose have been shown between commercial TG-43 software packages and MC simulations of TG-43 conditions. ${ }^{60}$ These discrepancies are attributable to differences in the assumed cross sections and dose-rate constants, and the treatment of sources as either points or lines.

\section{3 dtransform}

In radiotherapy when there is a dynamic geometry, dose mapping techniques are used to calculate the cumulative dose on the basis of dose distributions calculated on multiple anatomical instances of the patient. These techniques have been used in EBRT where large deformations due to breathing cycles or patient movement are not uncommon. $^{84}$

In this work, doses to transformed geometries were mapped to the reference geometry using dtransform, an energy mapping method $^{73}$ (figure 2.3). In this method, the voxels of the reference VPM are decomposed into tetrahedra which are warped to represent where the points of the tetrahedra would exist under the deformation. The code dtransform calculates the volume overlap of the warped tetrahedra with a voxel in the deformed geometry and the corresponding fraction of the energy deposited in the deformed voxel contributes to the energy deposited in the warped tetrahedra. ${ }^{73}$ While the warped tetrahedra are free to exist anywhere, in this work the same voxel grid is used for both reference and deformed geometries (only the 
identity of the medium in a voxel is changed during deformation, not the boundaries of individual voxels). Voxelization of the geometries leads to partial volume effects when the warped tetrahedra partially overlaps a voxel of a different medium than the reference voxel that the warped tetrahedra represents. To avoid mapping energy between voxels containing different media, a check for medium consistency was implemented. For example, if a tetrahedron corresponding to a voxel of prostate tissue in the reference geometry partially overlaps a voxel assigned as calcification in the deformed geometry, then contributions to the energy and mass from that volume of inconsistent media are ignored. Summing the energy deposited in voxels of the same medium that intersect any tetrahedra representing a reference voxel and dividing by the mass of the reference voxel gives a mapped dose for that reference voxel,

$$
D_{\text {ref }}=\frac{E_{\text {def }}\left(\frac{V_{r e f}}{V_{d e f}}\right)}{V_{\text {ref }} \rho_{\text {ref }}}
$$

where $D_{\text {ref }}$ is the dose to the reference voxel, $E_{d e f}$ is the energy deposited in the deformed voxel, and $V_{\text {ref }}$ and $V_{\text {def }}$ are the respective volumes of the reference and deformed voxels, as shown in figure 2.3. Multiplying by the ratio of volumes extracts the energy that was deposited in the tissue fraction of the deformed voxel. The dose to the edematic fluid does not contribute to the dose to the reference geometry so it is discarded.

\subsection{Edema modelling process}

Edema is modelled in the simulation geometries by applying an affine transform that expands the prostate about its geometric center and overwriting tissue that falls within the new contour to represent prostate tissue. Organs other than the prostate (rectum, bladder) are not deformed but may be overwritten in places where they are 


\section{Reference geometry Expanded geometry}
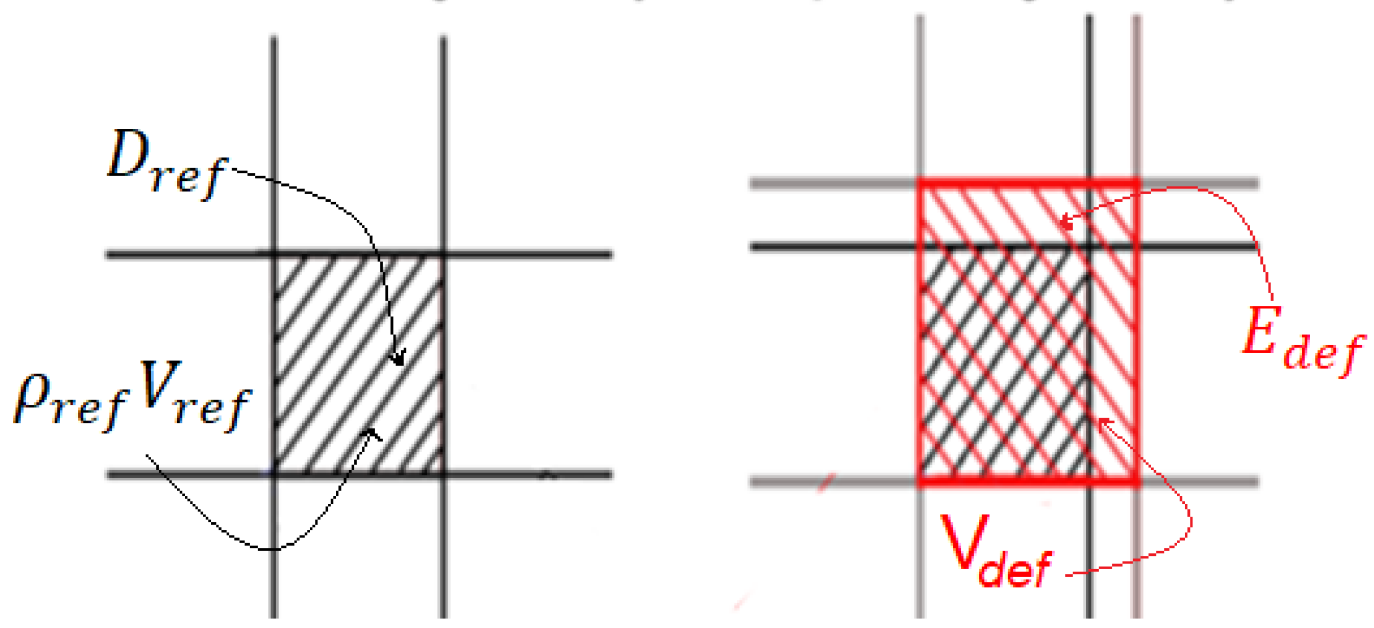

Figure 2.3: Simplified representation of the energy mapping process. The mapping technique decomposes each voxel into 6 tetrahedra. A complete voxel (rather than tetrahedra) is shown here for conceptual clarity. The black voxel-grid represents the grid used for all geometries. The grey voxel grid represents warped voxels.

overlapped by the new prostate. Bone is neither deformed nor overwritten. Rigid bodies (seeds, calcification) within the prostate are displaced consistently with the prostate tissue they are embedded in.

\subsubsection{Generating intermediate geometries with edema}

The CT-based VPM associates a medium and a density to each voxel. To expand the dimensions of the prostate in the VPM, voxels which are initially not prostate are reassigned as prostate tissue. Densities of non-prostate tissues are determined directly from the CT number for the voxel. All prostate voxels (including those from the initial CT-based VPM) are given a density equal to the nominal prostate density for that patient, defined as the average CT-number-derived density of all voxels identified as prostate in the reference VPM, for consistency with prostate voxels that are added in the process of generating an expanded geometry. Figure 2.4 shows that this approximation introduces a small discrepancy $(0.06 \%)$ in the calculated dose 

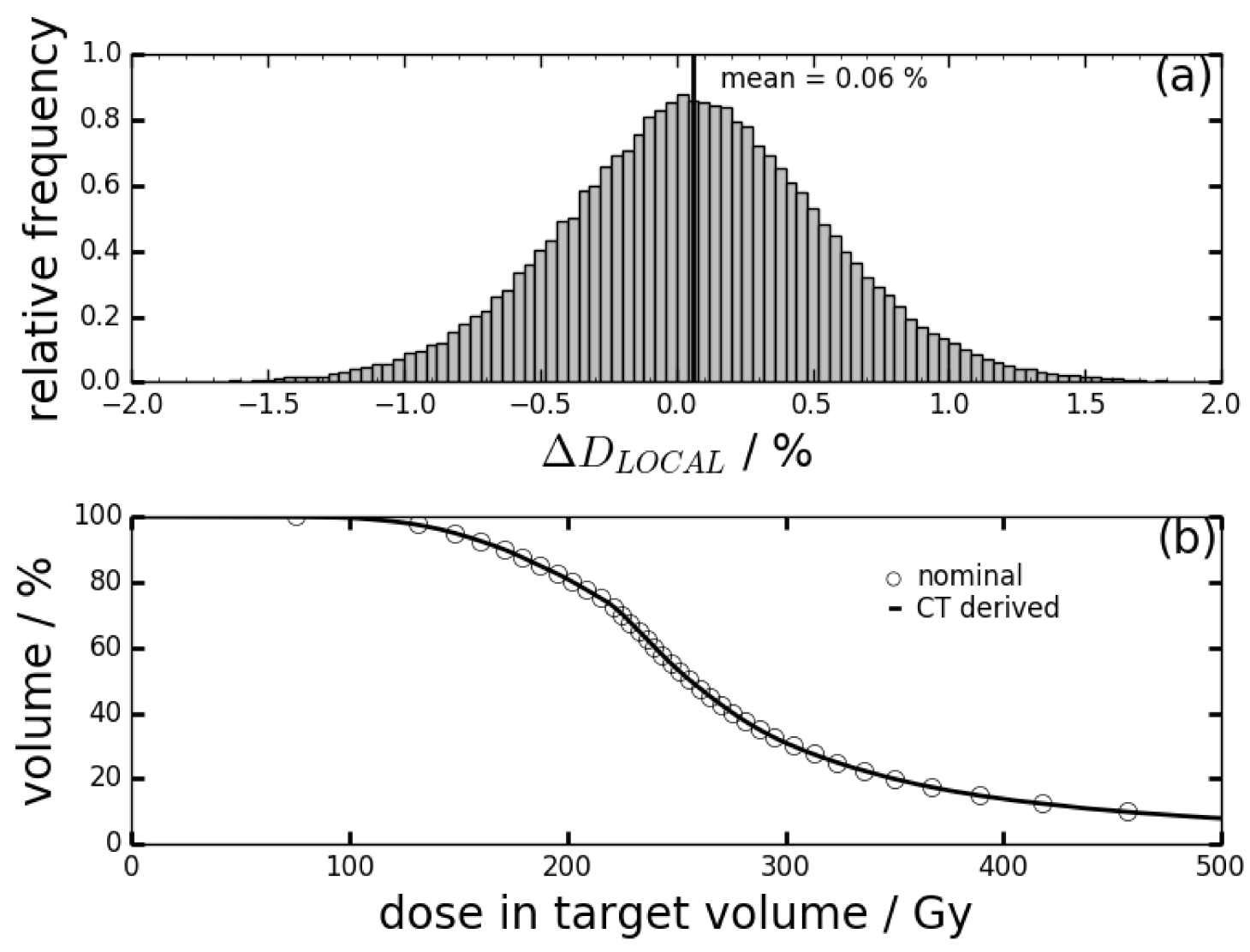

Figure 2.4: (a) Voxel-by-voxel comparison $\left(\Delta D_{L O C A L}\right.$, equation 2.3) of the dose to target tissue using the nominal density approximation or the raw CT-derived values, normalized by the dose calculated with the raw CT-derived values. (b) Dose-volume histogram comparison between doses calculated using nominal density (open circles) and raw CT-derived (solid) density values. The differences in calculated dose is negligible when calculating DVH metrics.

at the voxel scale, but this difference is negligible when considering DVHs.

From the reference VPM, a binary mask of the prostate constituents (prostate, calcification, $50 \%$ prostate $/ 50 \%$ calcification) is extracted and an affine transform is applied to the mask expanding it about its center using a standard scaling transform matrix, 


$$
\left[\begin{array}{cccc}
X_{1} & 0 & 0 & 0 \\
0 & X_{2} & 0 & 0 \\
0 & 0 & X_{3} & 0 \\
0 & 0 & 0 & 1
\end{array}\right]
$$

where $X_{j}$ is the expansion coefficient in the $j^{\text {th }}$ dimension. The inclusion of the fourth column allows the matrix to take the form of a homogeneous transformation matrix. The transformation is implemented for each point in the binary mask using the MATLAB ${ }^{85}$ function 'imwarp'.

With the transform defined independently in each of 3 dimensions, the displacement of a voxel can be simplified into a system of 3 identical independent 1dimensional equations

$$
m^{\prime}=m+\left\lfloor X_{m}\left(m-\odot_{m}\right)\right\rfloor,
$$

where $m^{\prime}$ is the index of the voxel of the shifted element, $m$ is the index of the voxel of the element, $X_{\mathrm{m}}$ and $\odot_{m}$ are the expansion coefficient and voxel position of the center of expansion, respectively, all for the $m^{\text {th }}$ axis. The voxel discretization necessitates that any translation be expressible as an integer number of voxels, so the floor $(\lfloor\rfloor$, rounding down to the nearest integer) is taken to ensure that at the edges of the prostate there are no 'island' voxels where rounding effects have caused one voxel to shift further than the immediate neighbours. Discretizing the voxel shift can therefore introduce discrepancies between the prescribed position and the modelled position by as much as the dimension of a voxel in the worst case. In the transverse plane this discrepancy is sub-millimeter, but on the inferior-superior axis the discrepancy can be larger because of lower image resolution on that axis.

After scaling the image, the coordinates of the prostate have been shifted. To correct for this the centroid is calculated for the initial and transformed masks and used to align the expanded mask to the VPM. The VPM is then modified so that 
any voxel falling within the bounds of the expanded prostate mask has the medium overwritten as prostate and the density is overwritten as the nominal prostate density. With the reference and expanded prostates in the same coordinate system, the displacement of an individual voxel in the transformation is given by equation (2.6), so the relationship between a point in the initial VPM and that point represented in the deformed VPM can be directly defined in a set of deformation vectors.

Rigid materials in the prostate (such as calcification or treatment seeds) are translated as defined by equation (2.6) as the prostate tissue expands. These rigid bodies do not expand, so each voxel corresponding to a rigid body in the reference VPM is represented by exactly one voxel in the deformed VPM; rigid body volume is conserved. To prevent merging of translated voxels as a result of discretization of the VPM, the neighbours of a calcified voxel are recursively searched to build a block of contiguous calcification. The appropriate shift is calculated for the centroid of the block and applied to each element of the calcification, resulting in a shift of the block as a unit, conserving both volume and shape. The procedure is identically repeated for voxels of $50 \%$ prostate $/ 50 \%$ calcification, and then both media types are overwritten into the transformed prostate.

Seed locations are defined externally to the VPM by a list of the positions of the center of each treatment seed and its orientation, which instructs egs_brachy where to position the source model in the simulation. While the location of the seed is represented as a point at the seed center, each seed is simulated as a detailed geometric model. In the process of modelling the treatment plans it was discovered that the clinically-determined seed positions occasionally resulted in an unphysical scenario of two seeds existing in the same space. This feature occurred at least once in each of the 120 treatment plans, for a total of 1057 conflicts over the 120 patients (mean $\approx 9$ per patient). This can be attributed to the uncertainties inherent in identifying seed positions from a CT image due to metallic artifacts and the discretization of a 
continuous volume into a series of two-dimensional image 'slices'.

To resolve this unphysical situation, pairs of conflicting seeds are translated in the transverse plane by the minimum amount necessary to achieve separation. In the case of multi-seed clusters, a shift that resolves a conflict between a pair of seeds could result in a new conflict with a third party seed. To ensure that full separation of all seeds is achieved, the open-source 3D modelling software Blender ${ }^{86}$ was utilized for intersection checking. Intersecting seed pairs are iteratively shifted until all conflicts are resolved.

The maximum displacement of a treatment seed to achieve separation across all treatment plans was 2.2 seed radii (figure 2.5) corresponding to just under a millimetre. To evaluate the magnitude of the error this introduces, it can be compared to the uncertainty in the determined seed location due to metallic artifacts. Depending on the chosen algorithm, metallic artifact corrections were applied to the CT images with a radius of $5 \mathrm{~mm}$ or five voxels (corresponding to a roughly $2 \mathrm{~mm}$ radius) depending on the algorithm, ${ }^{74}$ so the displacements applied to the seed locations do not exceed the uncertainty in seed position associated with metallic artifacts.

The $X, Y$ and $Z$ components of the location of a seed post-expansion can be determined

$$
k^{\prime}=k+X_{k}\left(k-\odot_{k}\right),
$$

where $k^{\prime}$ is the new coordinate, $k$ is the original coordinate, $\odot_{k}$ is the coordinate of the prostate centroid and $X_{k}$ is the expansion coefficient, all along the $k^{\text {th }}$ axis. Equation (2.7) is functionally the same as equation (2.6), except that egs_brachy does not require sources to be aligned with the discrete grid, so seed position can be calculated continuously. It was assumed that there is no rotation of the seed relative to the treatment plan and that the long axis of the seed will remain aligned with the needle which in the implant procedure is along the superior-inferior axis. Seed orientation does not significantly affect the calculated dose for the clinical treatment 


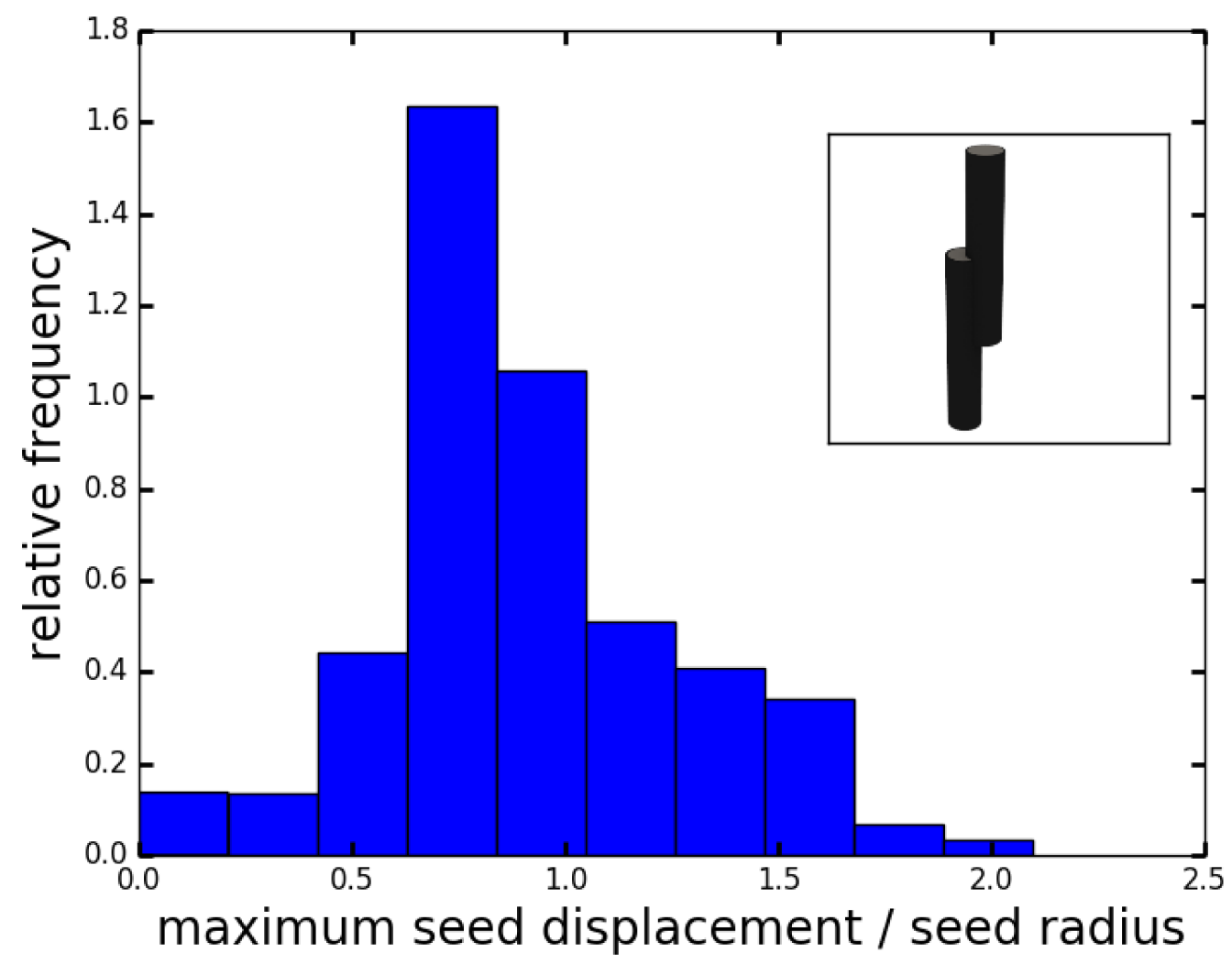

Figure 2.5: The maximum displacement from the clinically-determined seed locations required to resolve seed conflicts in each of 120 treatment plans, presented in terms of seed radii. The maximum displacement for any treatment plan is 2.2 radii. Inset: an example of intersecting seed models resulting from clinically-determined seed positions. 
volume in PIPB. ${ }^{63}$

\subsubsection{Dose mapping}

In addition to the reference and deformed geometries and the dose to the deformed geometry, dtransform requires a set of deformation vectors that describes the relationship between points in the two geometries in order to map the dose. Deformation vectors for each voxel in the mapped volume are calculated using equation (2.6) which represents the displacement of a voxel from the reference to the expanded geometry. The mapped volume includes all voxels within the prostate contour (prostate, calcification, $50 \%$ prostate $/ 50 \%$ calcification).

\subsubsection{Dose accumulation}

The dose to each transformed geometry is mapped onto the reference geometry. Every voxel of the target in the reference geometry has a 1:1 representation in each of the mapped geometries. As a result, the accumulated dose to a voxel $v$ can be calculated as a function of the dose to that voxel in each of the intermediate dose distributions:

$$
D_{v}^{a c c}=\sum_{j=1}^{n} D_{v}^{j} W_{j}
$$

where $D_{v}^{a c c}$ is the accumulated dose to the voxel $v, D_{v}^{j}$ is the dose to the voxel $v$ in the intermediate state $j$, and $W_{j}$ is the weighting factor applied to the intermediate state $j$ which is discussed below.

The weighting factor $W_{j}$ is the proportion of total source decays that occur while the actual geometry is represented by the transformed geometry $j$. The time during the treatment where $j$ is the active intermediate state depends on the edema model. The edema resolution model dictates the volume of the prostate at a given time, so from the volume of the prostate in each VPM, the time associated with that state is 
known, representing discrete points in the treatment time-course where the volume is exactly modelled. Figure 2.6 shows how the weight applied to each intermediate VPM is calculated. The intermediate state $j$ is taken to be the appropriate VPM until the fractional volume prescribed by the edema model is closer to the volume of next intermediate state $j+1$. For an edema model with an exponential resolution, the time at which the prescribed volume becomes closer to the volume of intermediate $j+1$ than the volume of intermediate state $j$ is given by

$$
t_{j}=\frac{t_{1 / 2}^{e}}{\ln 2} \cdot \ln \left[\frac{\Delta_{\max }}{\left(\frac{\Delta_{j}+\Delta_{j+1}}{2}\right)}\right]
$$

where $t_{j}$ is the time at which the intermediate $j$ stops being the appropriate model in favour of intermediate $j+1, t_{1 / 2}^{e}$ is the edema resolution half-life, $\Delta_{j}$ is the edema magnitude in the $j^{\text {th }}$ intermediate VPM and $\Delta_{\max }$ is the edema magnitude in the largest intermediate VPM as a fraction of the reference volume. These points define the interior boundaries of a set of 'bins' with two other points needed to complete the set: the first bin edge is located at time $t_{1}=0$ and the final bin edge is effectively located at time $t=\infty$.

In the case of a linear resolution model the time bin edges are defined

$$
t_{j}=\frac{\left[\Delta_{\max }-\left(\frac{\Delta_{j}+\Delta_{j+1}}{2}\right)\right] T_{f}}{1-\Delta_{\max }}
$$

where $T_{f}$ is the time at which the edema is completely resolved. Again, the first bin edge is at time $t=0$ and the last bin edge is $t=\infty$. The volume curve in Figure 2.6 is essentially decomposed into a step function of the most appropriate intermediate state $j$ for any given time $t$.

Integrating the time-step for intermediate $j$ under the activity curve gives the number of decays that occur while that intermediate is the active geometry model. 
Normalizing to the total number of decays gives the weighting factor $W_{j}$ for all but the final intermediate

$$
W_{j}=\lambda_{s} \int_{t_{j}}^{t_{j+1}} e^{-\lambda_{s} t} d t, j=1,2 . . n-1
$$

where $\lambda_{s}$ is the source decay constant and $t_{j}$ is a bin edge. The final intermediate state receives the remainder of the weighting

$$
W_{n}=1-\sum_{j=1}^{n-1} W_{j}
$$

In figure 2.6, dots on the edema curve represent the volume of the prostate in the available intermediate geometries. Vertical lines demarcate the time-bin for each intermediate, as defined in equation (2.9) to occur when the modelled volume is midway between 2 intermediate geometries. The time-bin is used as the bounds of integration under the activity curve for determining the proportion of decays that occur while an intermediate geometry is active and thus the dose-accumulation weighting.

\subsubsection{Time-course discretization}

The dose accumulation technique has not yet addressed how the number of intermediate geometries influences the accuracy of the accumulated dose. The use of more intermediate states yields a more accurate answer, but also increases the computational load, so practically it is useful to find the minimum number of intermediate states that will give a sufficiently accurate answer.

For isotropic expansions, the expansion coefficients $X_{j}$ are the same in each dimension. The expansion coefficients for intermediate geometries were selected to be evenly distributed with an interval $I_{X}$ (value to be determined), across the range of the linear expansion coefficient for that model. For an isotropic expansion the 


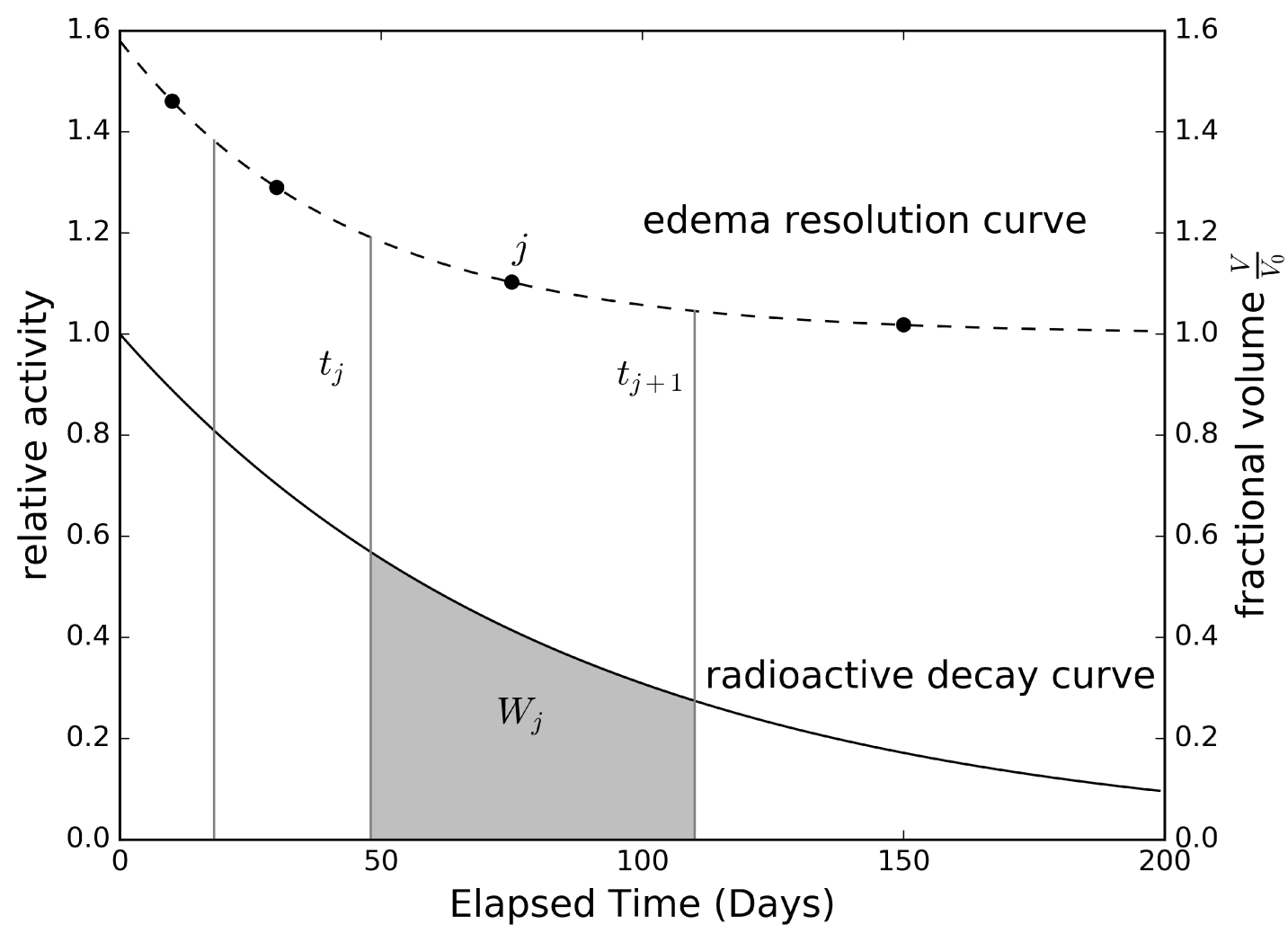

Figure 2.6: Graphic visualization of the determination of dose-accumulation weightings and how they depend on edema resolution model ( $c$, dashed line), the volumes of intermediate geometries (circles), and the decay of the source (solid line). Vertical lines indicate the edge of a time-bin. $t_{j}$ and $t_{j+1}$ indicate the bounds of integration when calculating $W_{j}$, the weighting factor for intermediate geometry $j$. 
maximum expansion coefficient is given by the third root of the maximum prostate volume (as a ratio of the reference volume, $\Delta_{\max }$ ), so that the maximum expansion coefficient for an edema model is

$$
X_{\text {max }}=\sqrt[3]{\Delta_{\max }} .
$$

For an exponential edema resolution, this is not a linear distribution with time. Instead, the geometries are more densely concentrated where the edema is highest which is also where the edema is changing fastest and a finer resolution is more beneficial.

For a subset of 3 patients with different \%IC, defined as the amount of intraprostatic calcification as a $\%$ by volume of the prostate, dose accumulation was performed using different intervals between expansion coefficient ranging from 5-100\% of $X_{\max }$. Dose metrics of the resulting dose distributions were then compared to the value of the metric calculated using the smallest interval. The appropriate interval $I_{X}$ was then evaluated as the interval

$$
I_{X}=\alpha \cdot X_{\max },
$$

where $\alpha$ is determined empirically such that for all considered metrics an accumulation using an interval $I_{X}$ the value of the metric is $<1 \%$ different than the value calculated by an accumulation with the finest resolution. A target discrepancy of $1 \%$ for all considered metrics was chosen because this value is comparable to the scale of other uncertainties inherent in PIPB dosimetry that will limit the accuracy, so further increasing the number of intermediate states accomplishes only an increased computational load.

\subsubsection{Dose evaluation}

As discussed in section 1.4.2, DVH-based metrics can be useful tools to evaluate dose distributions. The $\mathrm{D}_{x}$ family of DVH metrics can be determined as the dose 
to the $x \%+1^{\text {th }}$ lowest dosed voxel in the target. Whereas determining the $\mathrm{D}_{x}$ family involves finding the dose associated with a particular voxel, determining the $\mathrm{V}_{y}$ family represents the inverse problem of finding the number of voxels associated with dose. In practice, both types of metrics can be determined simply by sorting the voxel doses to voxels from lowest to highest and identifying the appropriate index; the $x \%+1^{\text {th }}$ voxel dose represents $\mathrm{D}_{x}$, and the proportion of the voxel doses that fall after the dose reaches $y$ indicates $\mathrm{V}_{y}$ (provided that voxels are of uniform volume). DVHs were created and metrics were extracted using the open-source code 3 ddose_tools. ${ }^{82}$

\subsubsection{Edema resolution models}

Ten clinically-observed patterns of edema were selected from the literature, ${ }^{37-42,68-70}$ representing the volume over time as the edema resolves. The resolution can be either exponential or linear with time. For use in the dose accumulation technique, each edema model must have an associated maximum edema and half-life (or the time for complete resolution in the case of a linear model). Several of the studies report only the relative volumes at specific time-points post-implant, without giving any half-life of the edema resolution. In these cases the model parameters were derived from two points assuming an exponential resolution as follows

$$
t_{\frac{1}{2}}^{e}=\frac{\left(T_{2}-T_{1}\right) \ln 2}{\ln \left(\frac{\Delta_{T 1}}{\Delta_{T 2}}\right)}
$$

where $t_{\frac{1}{2}}^{e}$ represents the half-life, $T_{1}$ and $T_{2}$ represent the time of each point, and $\Delta_{T 1}$ and $\Delta_{T 2}$ represent the volume of the prostate normalized by the reference volume at each time-point. The parameters of each edema model are summarized in table 2.3. 
Table 2.3: Parameters of edema resolution models from references indicated by labels used as shorthand for the remaining chapters: $\Delta_{\max }$ is the maximum edema, and $t_{1 / 2}^{e}$ is the edema resolution half-life.

\begin{tabular}{|c|c|c|c|c|}
\hline Label & Reference & $\Delta_{\max }$ & Half-life (days) & Shape \\
\hline $\mathrm{a}$ & ${ }^{\ddagger}$ Kovtun et al. ${ }^{68}$ & 1.24 & 12.5 & Exponential $^{\dagger}$ \\
\hline $\mathrm{b}$ & ${ }^{\ddagger}$ Kovtun et al..$^{68}$ & 1.33 & 5 & Exponential ${ }^{\dagger}$ \\
\hline $\mathrm{c}$ & Leclerc et al..$^{38}$ & 1.58 & 30 & Exponential \\
\hline $\mathrm{d}$ & Monajemi et al. ${ }^{37}$ & 1.20 & $28^{*}$ & Linear \\
\hline $\mathrm{e}$ & Tanaka et al. ${ }^{69}$ & 1.36 & 15 & Exponential $^{\dagger}$ \\
\hline $\mathrm{f}$ & Taussky et al..$^{42}$ & 1.31 & 11 & Exponential $^{\dagger}$ \\
\hline $\mathrm{g}$ & Tejwani et al. ${ }^{41}$ & 1.38 & 8.2 & Exponential $^{\dagger}$ \\
\hline $\mathrm{h}$ & Waterman et al. ${ }^{70}$ & 1.53 & $19^{\ddagger}$ & Exponential \\
\hline $\mathrm{i}$ & Waterman et al. ${ }^{39}$ & 1.52 & 9.3 & Exponential \\
\hline J & Westendorp et al. ${ }^{40}$ & 1.18 & 7 & Exponential $^{\dagger}$ \\
\hline ref & Static & 1 & & \\
\hline
\end{tabular}

$\dagger$ No characteristic shape reported. Half-life derived from volume at two times according to equation (2.15), assuming exponential shape $\ddagger$ Shape reported but half-life not reported

* For the linear case the 'half-life' is instead the time taken for complete resolution

note: Kovtun et al. identified 2 resolution patterns, one for patients with small $\left(<25 \mathrm{~cm}^{3}\right)$ prostate volumes and one for patients with large $\left(>25 \mathrm{~cm}^{3}\right)$ prostate volumes. In this work these models are applied indiscriminately to initial prostate volume. 


\section{Chapter 3}

\section{Results}

\subsection{Time-course discretization}

The sensitivity of the accumulated dose to the imposed time-course discretization is investigated with a subset of 3 patients with varying amounts of intraprostatic calcification characterized as \% IC, the percent of the volume of the total prostate volume occupied by calcification. Two models of clinically observed edema ( $b$ and $c$, representing the shortest and longest half-lives, respectively), were discretized with varying fineness of time resolution between intermediate states. While the use of more intermediates yields a more accurate answer it also increases the computational load.

Figure 3.1 shows the sensitivity of $\mathrm{D}_{90}$ to the discretization of the time-course. Model $c$ is more sensitive than model $b$ to changes in the discretization because the maximum edema magnitude is much larger (the largest of the implemented edema resolution models) which leads to a larger dosimetric effect. All investigated metrics converge to an agreement of $<1 \%$ for $\alpha=0.4$ (equation 2.14).

For the smallest $\Delta_{\max }$ (maximum edema magnitude) in the set of edema models, $I_{X}=0.4\left(\sqrt[3]{\Delta_{\max }}-1\right)=0.02$ so this is the maximum value for the interval between expansion coefficients of consecutive states, $I_{X}$, that can be used while still achieving $<1 \%$ uncertainty. To be conservative with the resolution, this interval is used for all calculations. 


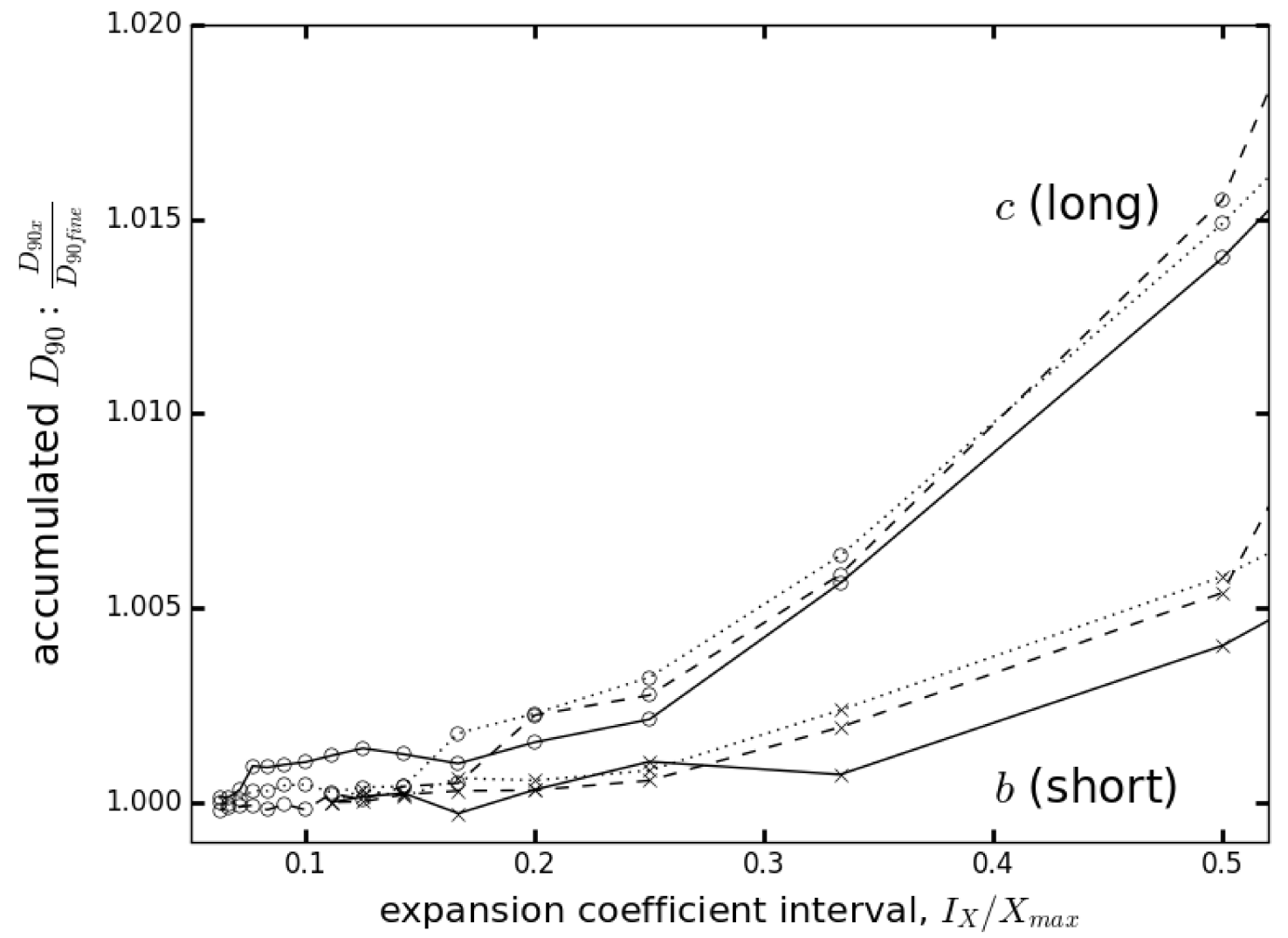

Figure 3.1: Investigating the sensitivity of calculated $\mathrm{D}_{90}$ to time-course discretization for edema models $b$ (short half-life, crosses) and $c$ (long half-life, circles) in 3 test patients; $0.02 \% \mathrm{IC}$ (solid), $0.59 \% \mathrm{IC}$ (dashed), and $3.8 \% \mathrm{IC}$ (dotted) where \% IC indicates the percent of the total prostate volume occupied by calcification. All metrics converged to within $1 \%$ of the finest resolution value for an expansion coefficient interval $I_{X}=0.4 X_{\max }$. 


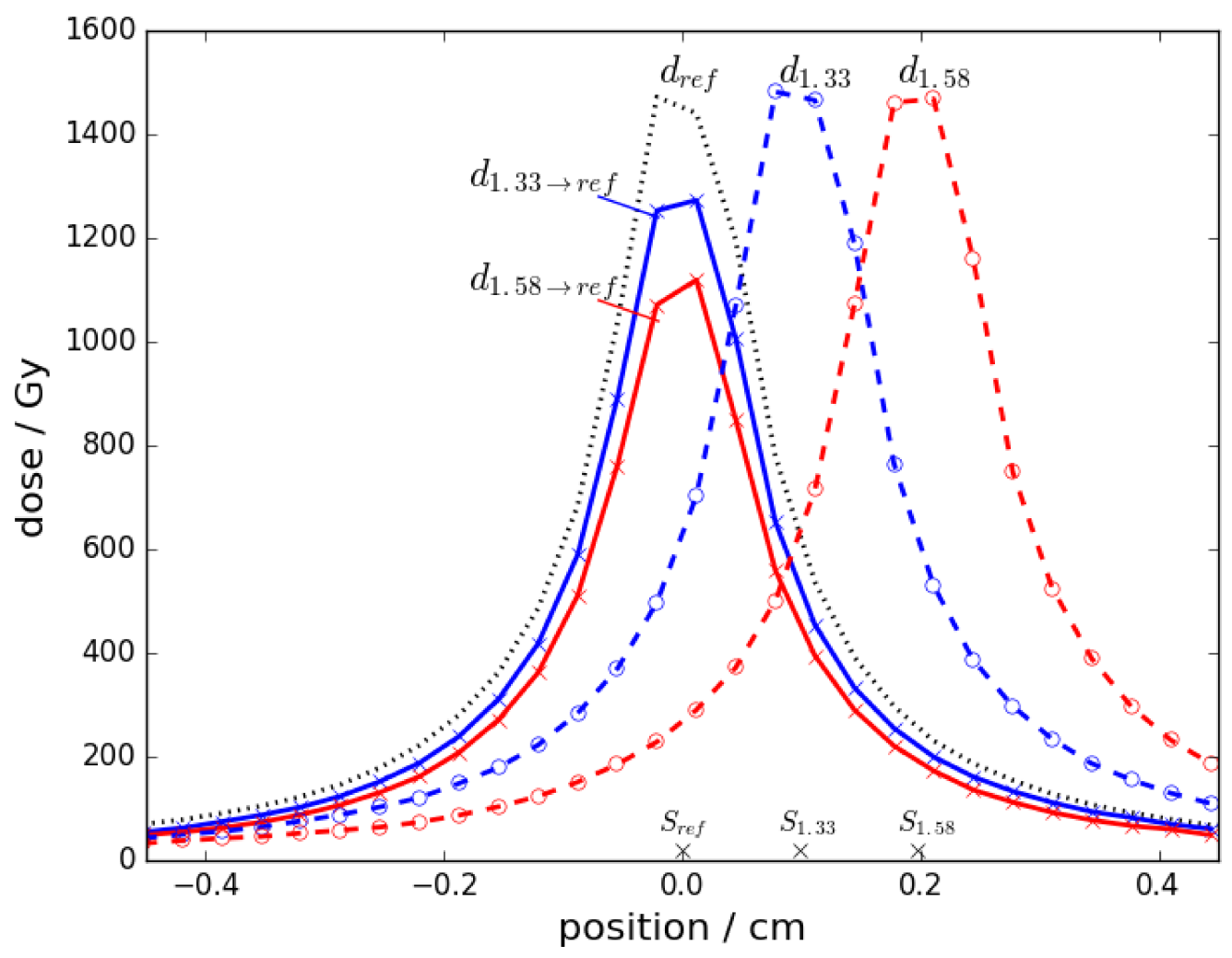

Figure 3.2: Dose profiles of the reference dose distribution $\left(d_{\text {ref }}\right.$; dotted $)$, dose distributions calculated using intermediate geometries with edema magnitudes of 1.33 and $1.58\left(d_{1.33}\right.$ and $d_{1.58}$, respectively) weighted as if they were the basis for a static calculation (dashed) and the intermediate dose distributions mapped to the reference geometry $\left(d_{1.33 \rightarrow \text { ref }}\right.$ and $d_{1.58 \rightarrow \text { ref }}$; solid). $S_{\text {ref }}, S_{1.33}$ and $S_{1.58}$ indicate the position of the seed centre along the axis in the subscripted geometry. In the mapped dose distributions the high-dose region is recovered at the location of the seed in the reference geometry.

\subsection{Analysis of dose mapping around a single seed}

The dosimetric effect of edema can be visualized neatly as a series of dose profiles near a treatment seed. Figure 3.2 shows the dose profile passing through the target with VPMs of increasing edema magnitude, and the same dose profile after the distribution has been mapped to the reference.

It can be seen in this figure that as the inter-seed distance increases (as $\Delta$ increases), the position of the high-dose regions associated with the seeds also moves. 
Mapping the dose distributions relocates the high-dose regions to the original seed positions in the reference VPM, as expected, but in the mapped dose distributions the dose decreases as the edema magnitude increases.

\subsection{Dose accumulation in example patients}

The following figures, figures 3.3, 3.4, and 3.5, each show the progression from VPMs to accumulated dose for 3 timepoints of the edema resolution: reference, moderate edema, and large edema. Each figure represents a different patient with varying \%IC (intraprostatic calcification), defined as the percent by volume of the prostate that is occupied by calcification. The accumulation model shown in these figures is model $c$ (see table 2.3), which is the model with the largest initial edema magnitude $\left(\Delta_{\max }=1.58\right)$. In addition to the dose distributions presented in the figures, clinical metrics are presented in table 3.1.

Figure 3.3 shows the progression from VPM to accumulated dose $\left(d_{r e f}^{a c c}\right)$ for a patient with $<0.01 \%$ IC. The edema time-course begins at the maximum magnitude, so the intermediate doses are arranged left to right, from largest to smallest edema magnitude so that the progression of time occurs left to right, but in investigating the effect of edema it is useful to compare to the reference dose distribution $d_{r e f}$. In the second row, note how for the intermediates with edema the dose distribution is 'pulled apart'. As the inter-seed separation increases, areas of the prostate that receive the prescription dose (145 Gy) in the reference distribution receive less than the prescription dose in the presence of edema. Mapping the dose to the reference geometry pulls some dose back towards the center of the prostate but there is still a reduction in the volume of the prostate covered by the prescription dose shown quantitatively in table 3.1. For an accumulation using edema model $c, \mathrm{~V}_{100}$ is reduced by $4 \%$ relative to a static $\mathrm{MC}$ dose calculation. 

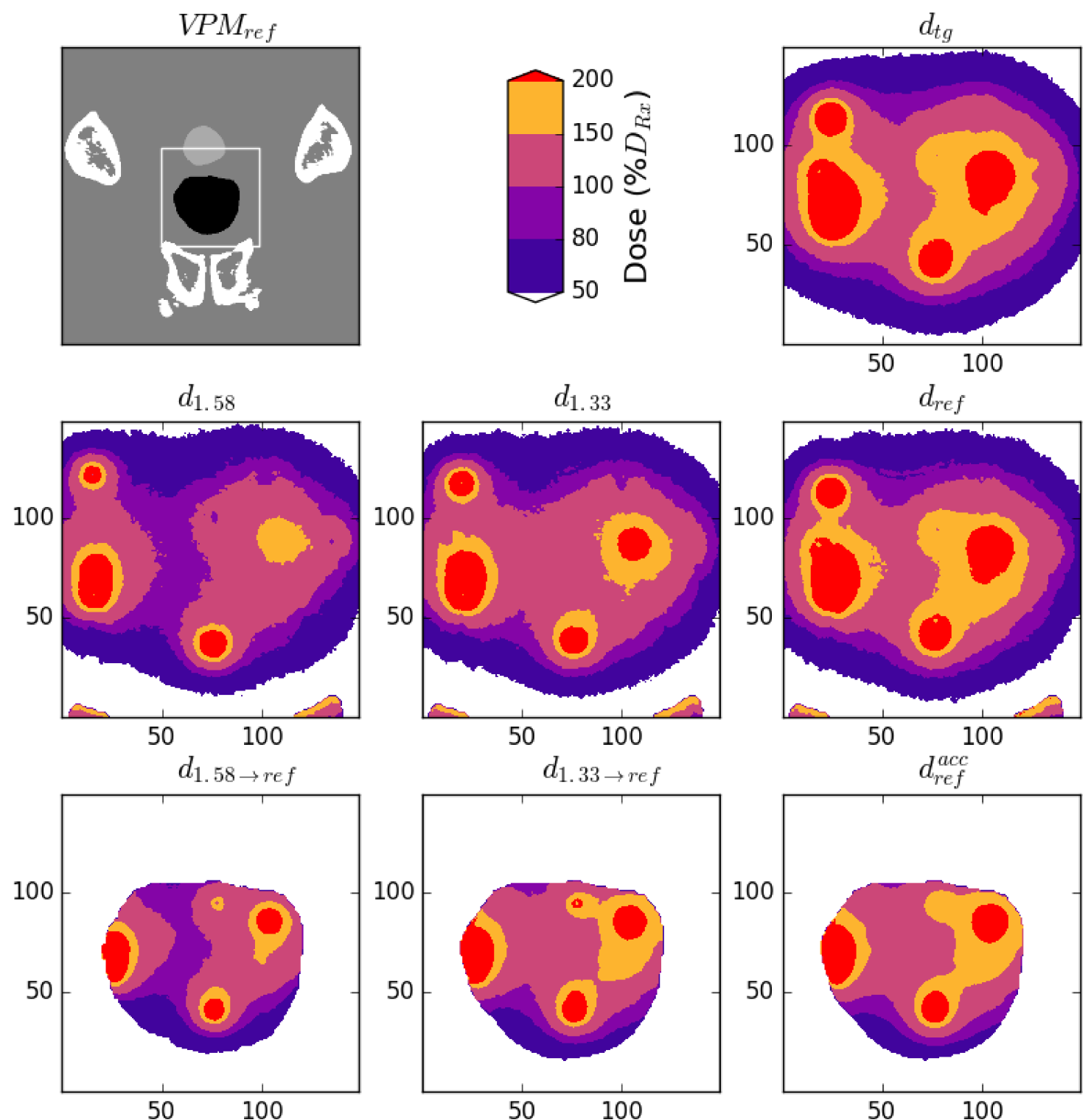

Figure 3.3: The evolution from virtual patient model (VPM) to accumulated dose for a patient with $<0.01 \%$ intraprostatic calcification by volume in the prostate. Top: the VPM with the prostate shown in black; the marked box indicates the area of the dose distributions shown in accompanying sub-figures. $\mathrm{d}_{t g}$ indicates the dose distribution calculated by the TG-43 formalism. Middle: Dose distributions calculated for edema magnitudes of 1.58, 1.33 and 1 (ref), weighted as if they were the basis for a static treatment. Bottom: Dose distributions mapped to the reference geometry and an accumulated dose distribution $\left(d_{r e f}^{a c c}\right)$ calculated assuming edema model $c$. A mapped dose is calculated only for those voxels within the prostate contour. Voxels beyond the prostate contour are shown as receiving no dose. 
Note in figure 3.3 that there is a seed implanted at approximately $(50,150)$, which the mapped sub-figures show is outside of the bounds of the prostate because dose is only mapped for those voxels that are within the prostate contour. Dose originating from this seed that is deposited in the prostate does still contribute to the mapped/accumulated dose distribution, but the associated very high dose region does not show in the mapped dose distribution sub-figures as an artifact of mapping the dose only for voxels in the target.

Figure 3.4 is similar to figure 3.3, however there is a small calcification in the lower left corner of the image of the prostate. The calcification can be recognized in the MC calculated dose distributions by the associated high dose region. It is distinguishable from a seed by comparing the MC dose distributions to the TG-43 calculated distribution. The TG-43 formalism ignores all changes in tissue composition, so the calcification does not perturb the smooth dose falloff in the TG-43 distribution as it does in the MC calculated distributions. As in the previous figure it can be seen that the increasing edema magnitude is associated with a lower dose delivered to the center of the prostate, however the dose to the center of the prostate in this slice was initially high enough that the reduction does not result in the dose falling below prescription in this slice of the accumulated dose, $d_{r e f}^{a c c}$. Over the entire prostate volume, however, the volume receiving a dose $>145$ Gy is reduced by $4 \%$ as seen in table 3.1 .

Figure 3.5 shows the accumulation process for a patient with multiple large calcifications. Note in the slice of the reference dose distribution, $d_{r e f}$, that the leftmost calcification is associated with a cold-spot due to the calcification shadowing the tissue from a nearby treatment seed. The same general trends are seen as in the previous patients; edema reduces the volume receiving a dose $>145$ Gy. For edema model $c$, $\mathrm{V}_{100}$ is reduced by more than $14 \%$ compared to a static MC dose calculation (table 3.1).

Based on a limited sample size of 3 patients it appears that the reduction in $V_{100}$ 

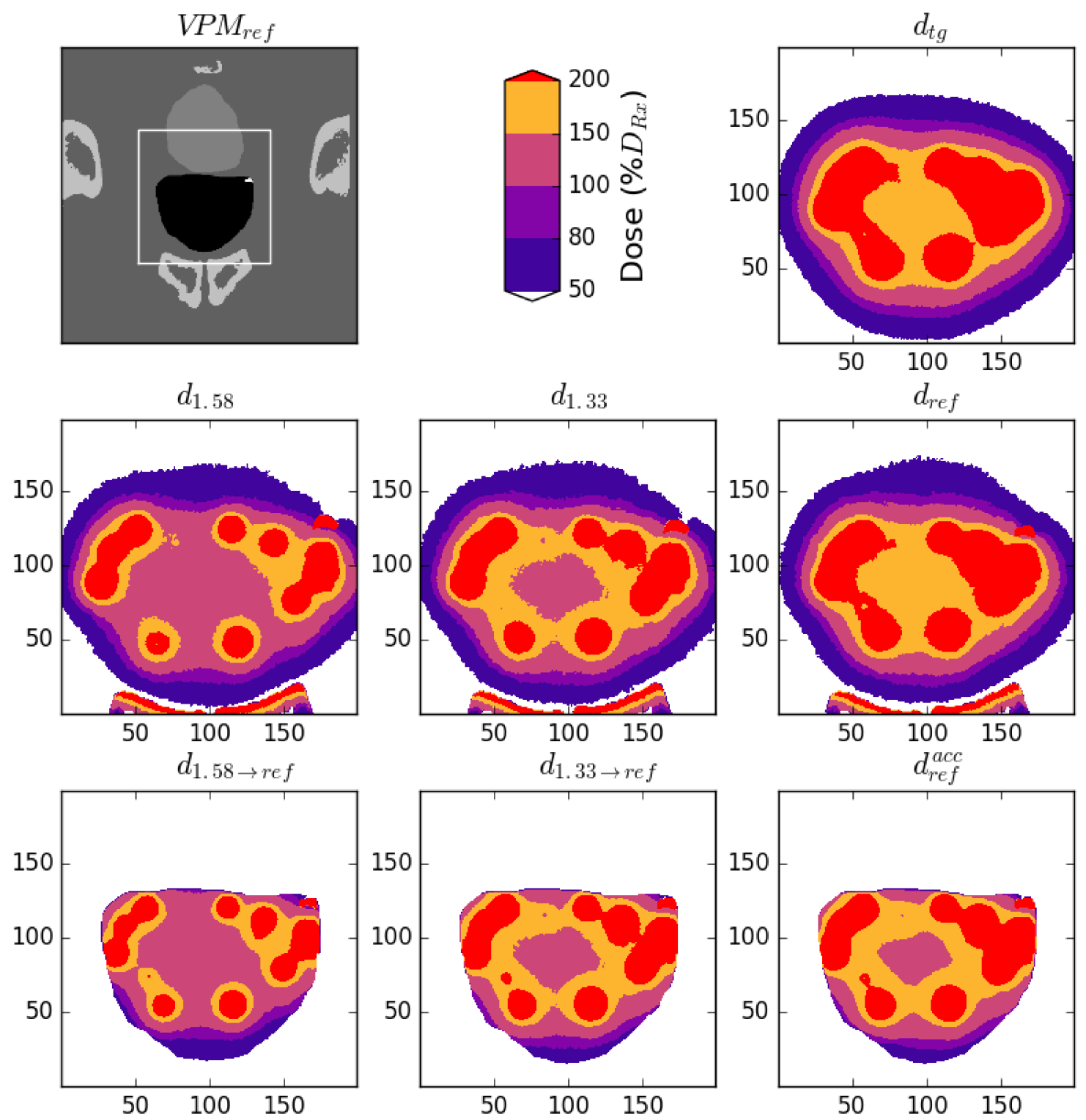

Figure 3.4: The same as figure 3.3 except for a patient with $0.33 \%$ (by volume) intraprostatic calcification. Calcification appears as a white patch on the upper-right corner of the image of the prostate. 

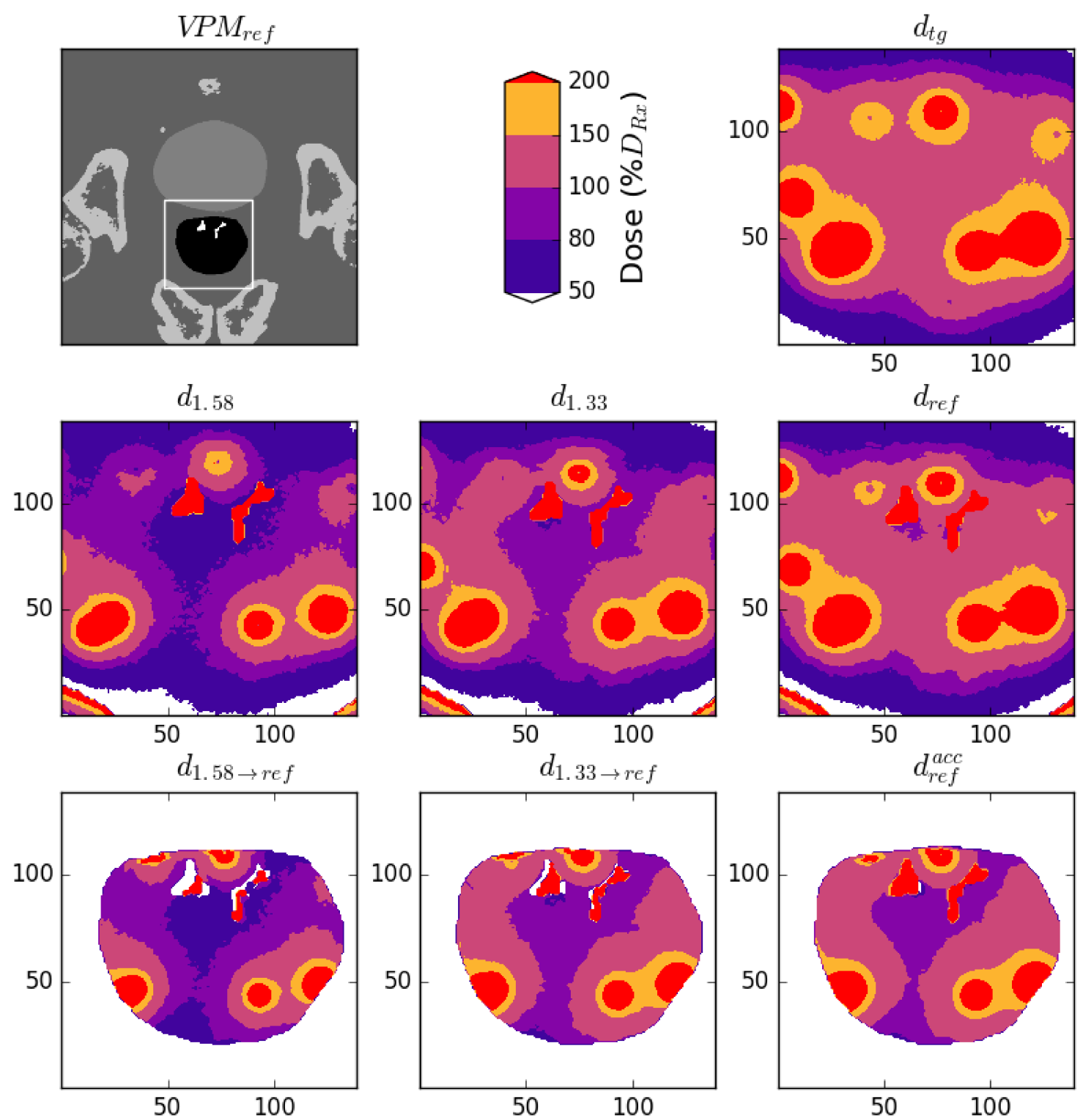

Figure 3.5: The same as figure 3.3 except for a patient with $2.2 \%$ (by volume) intraprostatic calcification. Calcification appears as white patches in the upper half of the image of the prostate. 
Table 3.1: Effect of edema on $\mathrm{D}_{90}, \mathrm{D}_{99}, \mathrm{~V}_{100}$ and $\mathrm{V}_{200}$ when adopting model-based dose calculation algorithm techniques for 3 representative patients and sub-cohort (IC: with intraprostatic calcification; NC: no intraprostatic calcification) averages.

\begin{tabular}{llccc}
\hline Patient Group & Metric & $d_{T G 43}$ & $d_{\text {ref }}$ & $d_{r e f}^{a c c}($ model $c)$ \\
\hline \hline \multirow{3}{*}{ Patient A } & D90 (Gy) & 166.9 & 159.8 & 141.7 \\
0.01\%IC & D99 (Gy) & 53.3 & 49.6 & 46.8 \\
& V100 & $94.1 \%$ & $93.1 \%$ & $89.1 \%$ \\
& V200 & $27.4 \%$ & $24.2 \%$ & $17.2 \%$ \\
\hline \multirow{5}{*}{ Patient B } & D90 (Gy) & 181.4 & 170.5 & 150.6 \\
0.33\%IC & D99 (Gy) & 98.3 & 76.0 & 72.9 \\
& V100 & $97.2 \%$ & $95.5 \%$ & $91.5 \%$ \\
& V200 & $39.4 \%$ & $34.2 \%$ & $24.1 \%$ \\
Patient C & D90 (Gy) & 143.9 & 125.8 & 107.2 \\
$2.2 \%$ IC & D99 (Gy) & 71.9 & 64.9 & 29.8 \\
& V100 & $89.7 \%$ & $81.0 \%$ & $66.6 \%$ \\
& V200 & $17.4 \%$ & $17.4 \%$ & $12.9 \%$ \\
\hline \multirow{5}{*}{ NC sub-cohort } & D99 (Gy) & 81.0 & 72.5 & 40.0 \\
(average) & V100 & $90.3 \%$ & $88.2 \%$ & $79.4 \%$ \\
& V200 & $33.6 \%$ & $30.1 \%$ & $21.7 \%$ \\
\hline \multirow{5}{*}{ IC sub-cohort } & D99 (Gy) & 76.4 & 67.3 & 39.3 \\
(average) & V100 & $89.9 \%$ & $87.0 \%$ & $77.8 \%$ \\
& V200 & $32.8 \%$ & $29.3 \%$ & $21.5 \%$ \\
\hline \hline
\end{tabular}




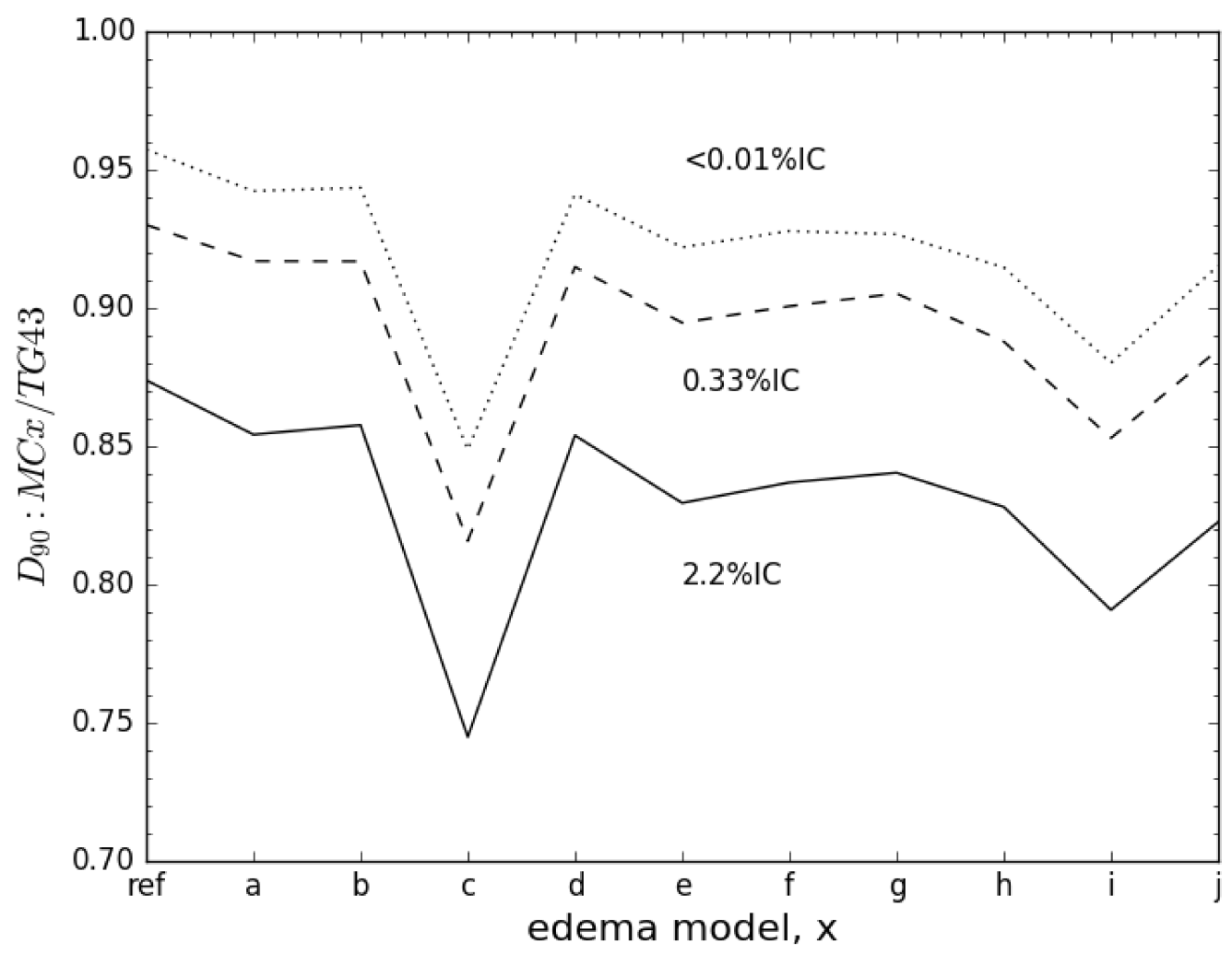

Figure 3.6: $\mathrm{D}_{90}$ from a static (ref) or accumulated $(a-j) \mathrm{MC}$ calculation divided by the TG-43 calculated $D_{90}$ value, for 3 patients with varying amounts of intraprostatic calcification. Lines connecting the points do not imply a relationship but are drawn only to guide the eye.

associated with edema has a dependence on the amount of calcification. The trend of greater $\mathrm{V}_{100}$ reduction for increasing \% IC holds at the sub-cohort level (with or without calcification), with a greater reduction in $\mathrm{V}_{100}$ relative to a static $\mathrm{MC}$ dose calculation when incorporating edema in patients in the IC (intraprostatic calcification) sub-cohort (9.2\%) than in patients in the $\mathrm{NC}$ (no calcification) sub-cohort $(8.8 \%)$.

Figure 3.6 shows that the relative dosimetric effect of each edema model is consistent across patients including those with different \%IC. The edema model that results in the largest dose reduction relative to the other edema models (edema model $c$ ) in one patient also results in the largest dose reduction in all other patients. The first 
point of each series in the figure also shows that the TG- 43 overestimation of $\mathrm{D}_{90}$ compared to a static MC dose calculation increases as \%IC increases, reaching almost 15\% of the calculated value for a patient with $2.2 \% \mathrm{IC}$.

Table 3.1 supports a correlation between $\mathrm{D}_{90}$ overestimation and \% IC for the sub-cohorts. For patients in the NC sub-cohort, including tissue composition in an MBCDA calculation reduces the calculated value of $\mathrm{D}_{90}$ by $8.1 \mathrm{~Gy}$, on average. For patients in the IC sub-cohort $\mathrm{D}_{90}$ is reduced by more than $10 \mathrm{~Gy}$.

\subsection{Full-tissue dynamic MC compared to TG-43}

Figure 3.7 shows the ratio of the $\mathrm{D}_{90}$ values calculated using MC models relative to the TG-43 value for 120 patients. The first pair (labelled 'ref') shows that simply by incorporating non-water tissues, the calculated $\mathrm{D}_{90}$ has fallen to less than $95 \%$ of the TG-43 value, and the effect appears more prominent in patients with calcification. The inter-group variability illustrates that the choice of edema model is an important factor to the final results, further reducing $\mathrm{D}_{90}$ by an additional $2-15 \%$ of the TG- 43 value. Model $c$ exhibits the greatest dosimetric effect. Model $c$ represents both the largest edema magnitude and the longest half-life of the utilized edema models. Total reduction in $\mathrm{D}_{90}$ (by both tissue composition and edema effects) relative to TG-43 can range from a few percent to $20 \%$. The magnitude of the reduction in $\mathrm{D}_{90}$ is dependent on the amount of calcification. $\mathrm{D}_{90}$ reductions from tissue composition and edema effects are systematically larger for patients in the IC sub-cohort than those in the NC sub-cohort.

Figure 3.8 is similar to figure 3.7 , except that it shows $\mathrm{D}_{99}$ (the minimum dose received by $99 \%$ of the target volume) rather than $\mathrm{D}_{90}$. The $\mathrm{D}_{99}$ metric exhibits similar trends to the $\mathrm{D}_{90}$ metric. TG-43 systematically overestimates the delivered $\mathrm{D}_{99}$ compared to a static $\mathrm{MC}$ calculation ('ref'), and the magnitude of this overestimation 


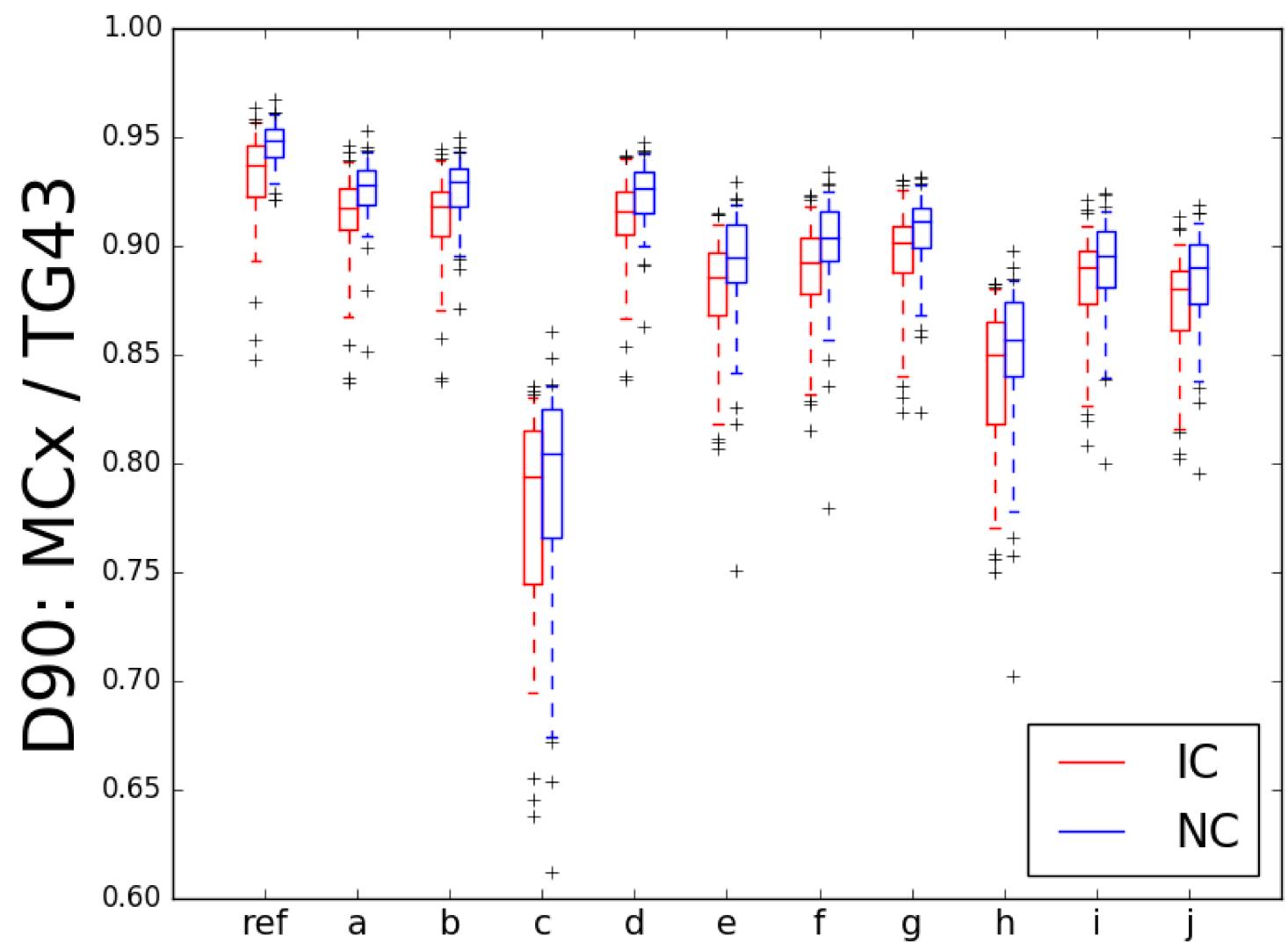

Figure 3.7: Box and whisker plot showing interquartile range (box), median (line) and $95^{\text {th }}$ percentiles (whiskers, + for outliers) of $\mathrm{MC}$ calculated $\mathrm{D}_{90}$ divided by the static TG-43 calculated value for ref (no edema) and edema models $a-j$ (table 2.3) for patients in the IC (intraprostatic calcification, $>0.3 \%$ by volume) and $\mathrm{NC}$ (no calcification) sub-cohorts consisting of 60 patients each. 


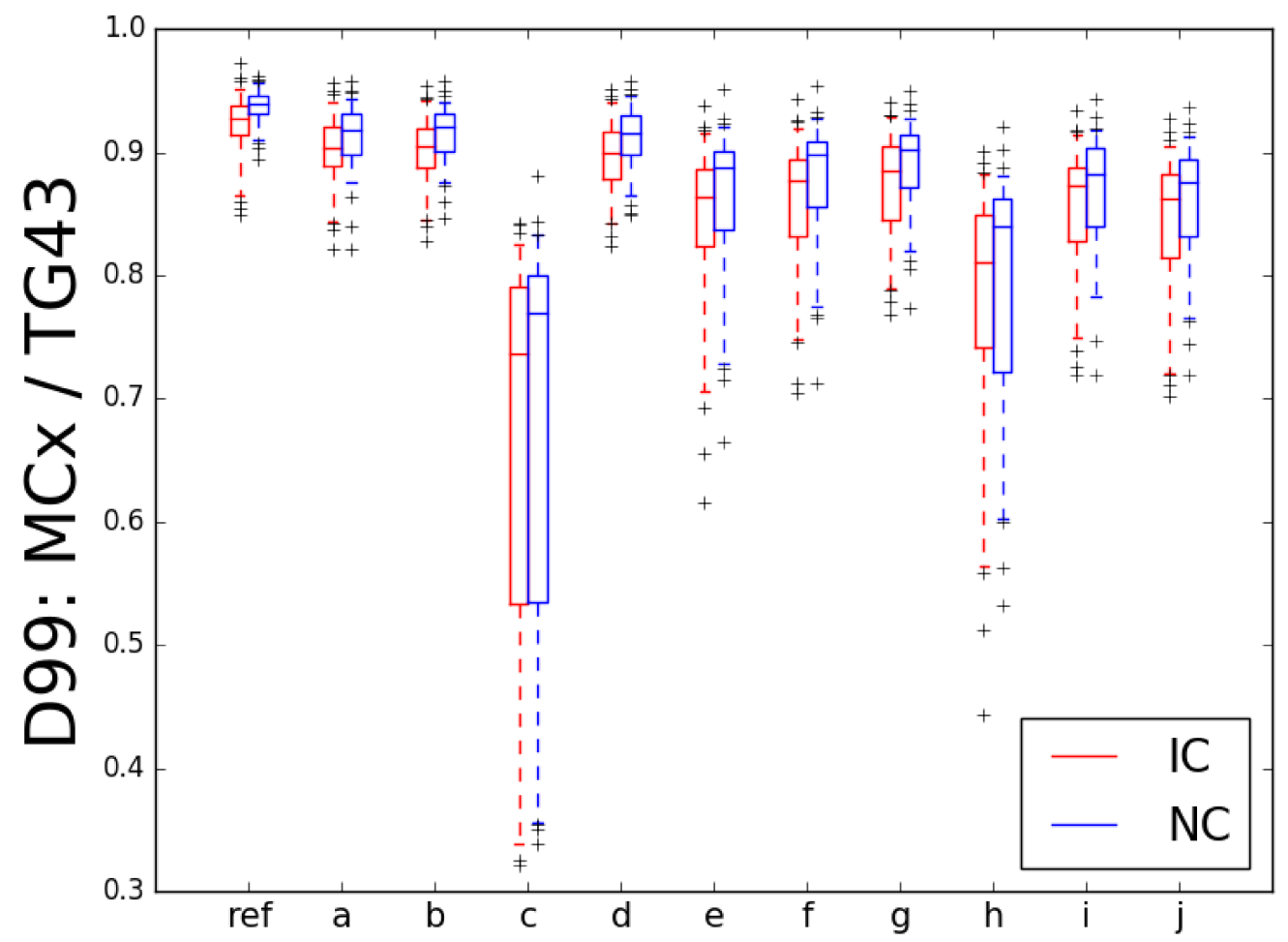

Figure 3.8: Box and whisker plot showing interquartile range (box), median (line) and $95^{\text {th }}$ percentiles (whiskers, + for outliers) of MC calculated $\mathrm{D}_{99}$ divided by the static TG-43 calculated value for ref (no edema) and edema models $a-j$ (table 2.3) for patients in the IC (intraprostatic calcification, $>0.3 \%$ by volume) and $\mathrm{NC}$ (no calcification) sub-cohorts consisting of 60 patients each. 
is larger for patients in the IC sub-cohort compared to those in the NC sub-cohort. Applying an edema model results in further reduction of $\mathrm{D}_{99}$ with the magnitude of the reduction depending on the edema model, ranging from $2-20 \%$ of the TG-43 calculated value. The very large reductions are seen for the edema model $c$. The magnitude of the reduction in the calculated $\mathrm{D}_{99}$ is systematically larger for patients in the IC sub-cohort compared to patients in the NC sub-cohort.

\subsection{Dynamic MC compared to static MC}

In the previous section it was shown that the values of the dose metrics $\mathrm{D}_{90}$ and $\mathrm{D}_{99}$ are decreased both by incorporating tissue composition variation and edema into the simulation. To evaluate the magnitude of the dosimetric effect of edema for these metrics, the value of the calculated metric for an edema-modelled dose distribution is normalized to the static MC calculated value to remove the presence (or lack of) tissue composition variation as a confounding effect.

Figure 3.9 shows the effect of edema on $\mathrm{D}_{90}$ for each edema model. Depending on the edema model, edema reduces the value of $\mathrm{D}_{90}$ by $1-15 \%$ of the static $\mathrm{MC}$ calculated $\mathrm{D}_{90}$ value. Note the mean value of the ratio for the IC sub-cohort is systematically slightly higher than for the NC sub-cohort. A higher value of the ratio indicates that the change in the metric due to edema is less.

Figure 3.10 shows the effect of edema on $\mathrm{D}_{99}$ for each edema model. Edema reduces the value of $\mathrm{D}_{99}$ by 1-20\% of the static MC calculated value on average, depending on the edema model. The magnitude of the effect of edema on the calculated $\mathrm{D}_{99}$ value appears to be slightly systematically less for patients in the IC sub-cohort compared to the NC sub-cohort. Note that for some statistical outliers in figure 3.10, the effect of edema is to increase the value of $\mathrm{D}_{99}$. 


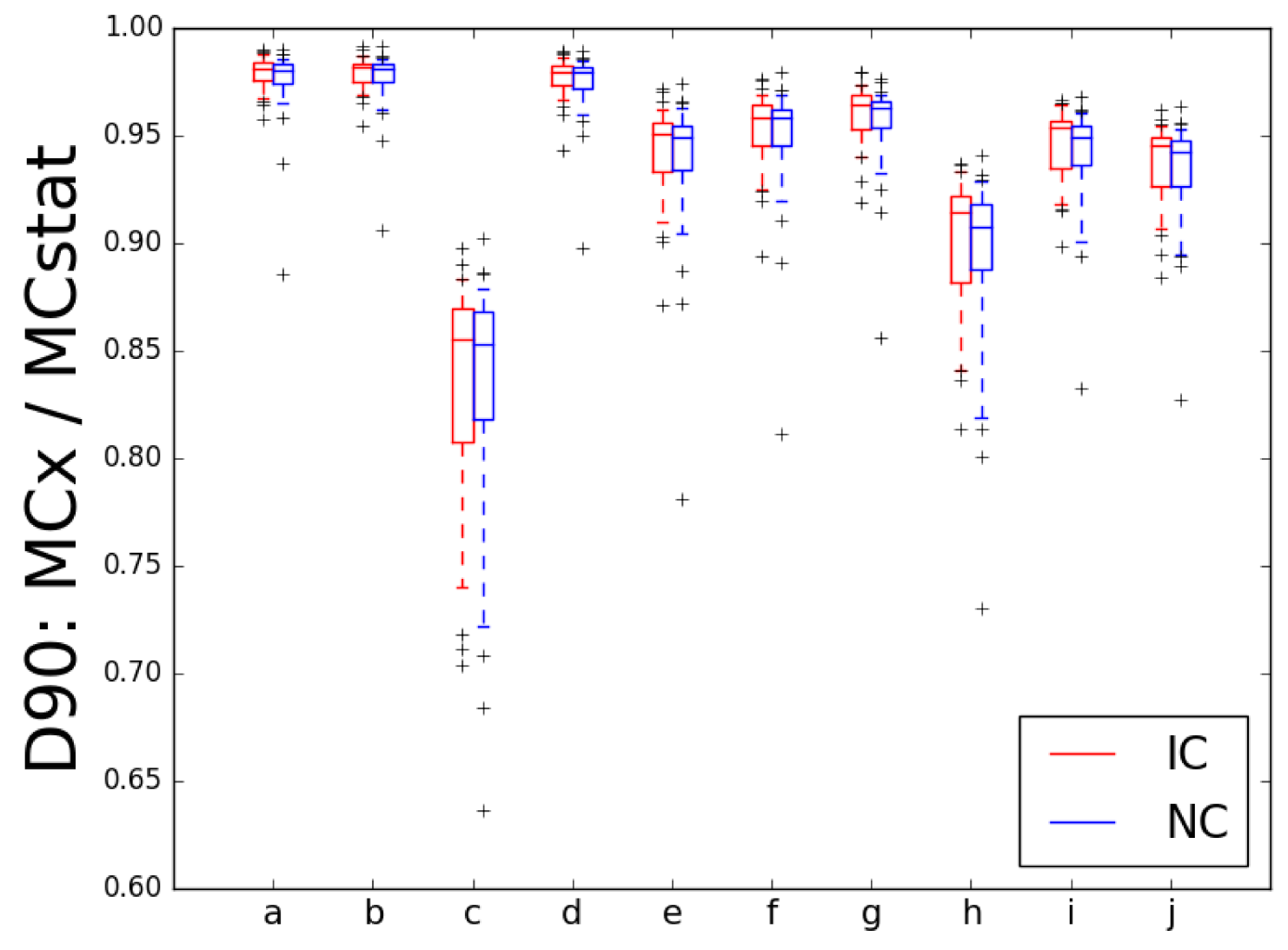

Figure 3.9: Box and whisker plot showing interquartile range (box), median (line) and $95^{\text {th }}$ percentiles (whiskers, + for outliers) of $\mathrm{D}_{90}$ divided by the static MC calculated D90. Edema models $a-j$ (table 2.3) for patients in the IC (intraprostatic calcification, $>0.3 \%$ by volume) and $\mathrm{NC}$ (no calcification) sub-cohorts consisting of 60 patients each. 


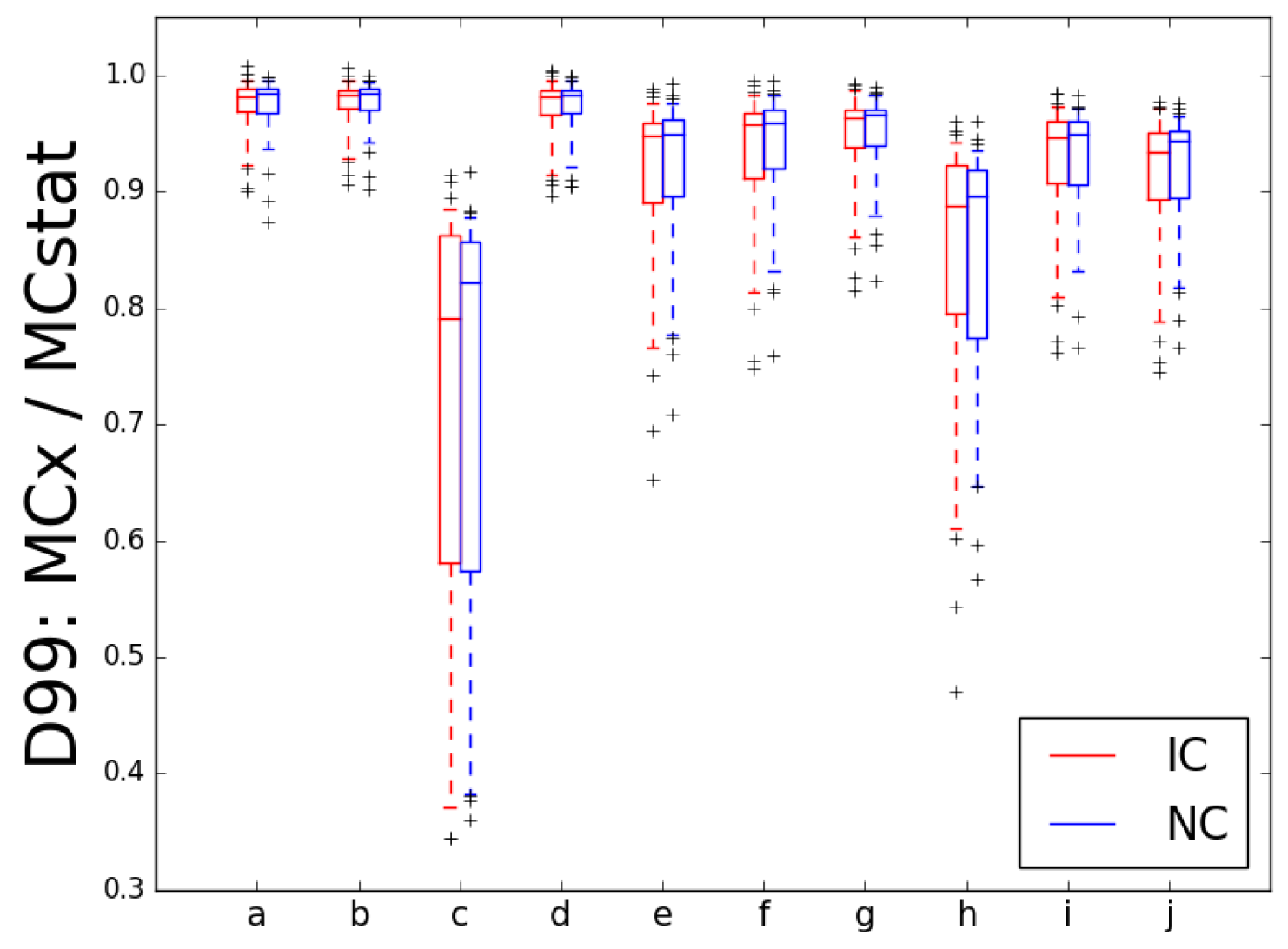

Figure 3.10: Box and whisker plot showing interquartile range (box), median (line) and $95^{\text {th }}$ percentiles (whiskers, + for outliers) of $\mathrm{D}_{99}$ divided by the static $\mathrm{MC}$ calculated $\mathrm{D}_{99}$ for ref (no edema) and edema models $a-j$ (table 2.3) for patients in the IC (intraprostatic calcification, $>0.3 \%$ by volume) and $\mathrm{NC}$ (no calcification) sub-cohorts consisting of 60 patients each. 


\section{Chapter 4}

\section{Discussion and conclusions}

\subsection{Discussion}

A framework was successfully implemented to calculate accumulated doses for prostates exhibiting edema over the course of a PIPB treatment using model-based dose calculation methods. Compared to the current clinical standard, TG-43, the accumulated dose distributions were systematically lower across the entire cohort. There were two major factors that affected the final calculated dose: the effect of edema and the effect of using a model that incorporates tissue composition (in contrast to the all-water TG-43 approach).

Existing TG-43 based approaches to modelling edema ${ }^{38,43,46-49}$ fail to make use of the available tissue-based information and as a result overestimate dose delivered to a static geometry by about $6 \%$ on average compared to a MC dose calculation (see table 3.1). A $6 \%$ reduction in $\mathrm{D}_{90}$ when comparing a static $\mathrm{MC}$ calculation to the TG-43 determined value is comparable to values found in the literature ${ }^{53,54}$ including a previous analysis of the entire 613 patient database by Miksys et al. ${ }^{56}$ There is a small disagreement in the values of the average dose metrics between this work and the work by Miksys et al. but this is expected as this study used only a subset of the complete dataset of 613 patients.

MC dose calculations of $\mathrm{PIPB}^{53,54,56,74}$ can incorporate tissue composition information, but as of yet has not been used to tackle the edema problem with the 
exception of one previous work by Mountris et al. ${ }^{45}$ Mountris and colleagues simulated, along with a range of edema models spanning a reasonable parameter space, the clinically observed ${ }^{41}$ edema resolution corresponding to model $g$ in this work. For this model, present in both works, Mountris et al. report that for a 15-patient cohort the average reduction in $\mathrm{D}_{90}$ was $22 \%$ for edema model $g$ compared to a static $\mathrm{MC}$ calculation, but we found that $\mathrm{D}_{90}$ the edema model $g$ reduced $\mathrm{D}_{90}$ by $12 \%$ of the static MC value. The method of generating intermediate geometries is a major difference between these works. Mountris et al. used a finite element approach to modelling fluid dynamics in order to predict the prostate geometry, in contrast to the method presented in this work of directly modifying VPMs to match a prescribed volume. There are likely differences in the method used to map doses before accumulation, and possibly differences in the dose accumulation algorithm (e.g., calculation of weights) but these cannot be properly evaluated because the techniques used by Mountris et al. are incompletely described.

A simultaneous examination of table 2.3 and the figures of section 3.4 shows that a larger or longer lasting edema is associated with greater reductions in dose. The edema models that exhibit the least dose reduction, $a$ and $b$, are the models with the smallest edema magnitude and half-life, respectively. The edema model that consistently exhibits the greatest reduction in dose, $c$, has both the largest edema magnitude and the largest edema half-life of the set of edema models. The larger the edema magnitude becomes, the less the actual geometry is represented by the planning geometry and the less successful the treatment plan becomes at adequately dosing the target region.

The major contributor to this edema magnitude dependence is simply a geometric relationship. As the target volume increases the distance from a point in the target to a seed also increases, and there is also a screening effect whereby the influx of interstitial fluid responsible for the swelling ${ }^{87}$ absorbs energy that otherwise would 


\section{CHAPTER 4. DISCUSSION AND CONCLUSIONS}

have been deposited in the target. The dependence on the edema resolution half-life is increased by an interplay with the radionuclide half-life. ${ }^{46,50,88}$ The greater the proportion of total decays in the treatment that happen while the target is swollen, the less like the treatment plan the resulting accumulated dose distribution will be. For a short edema resolution with a long-lived radionuclide, the edema could be almost completely resolved and reasonably well represented by $\mathrm{VPM}_{r e f}$ before the majority of source activity has been depleted. Recall that the edema parameters used in this work are the average parameters from studies in the literature, so an individual patient could have a larger or longer edema which would be associated with even greater dose reductions. Considering the above described dynamics of edema and radionuclide half-lives, the dosimetric effect of edema is even greater with a radionuclide source with a shorter half-life, for example ${ }^{103} \mathrm{Pd}$ with a half-life of 17 days ${ }^{50}$ or ${ }^{131}$ Cs with a half-life of 10 days. ${ }^{46,88}$

The relative importance of edema and tissue composition is dependent on the patient geometry. Using a full-tissue VPM for static dose calculations rather than TG-43 has a larger effect, on average, on $\mathrm{D}_{90}$ for those patients in the IC sub-cohort (10.3 Gy) compared to those in the NC sub-cohort (8.1 Gy), which is not surprising considering that the TG-43 water assumption is a worse approximation for the higher $\mathrm{Z}$ calcification than for normal prostate tissue. For the edema model with the largest dosimetric effect $\mathrm{D}_{90}$ is reduced from the TG-43 value by 33 Gy on average for both NC and IC patients. Section 1.4.3 discussed how $\mathrm{D}_{90}$ is an indicator of long-term outcomes and a $\mathrm{D}_{90}$ of less than the prescription dose is associated with reduced rates of freedom from biochemical failure. In this way, edema could contribute to interpatient variation in response to PIPB. While edema can cause large dose reductions throughout the target volume, the tissue composition effect reduces dose globally in the target volume but can also result in very large changes, either increasing or decreasing, to the local dose to a voxel. 


\section{CHAPTER 4. DISCUSSION AND CONCLUSIONS}

There are several important limitations that must be acknowledged in the application of the framework used here, stemming from the dose calculation algorithms and the creation of models of the patient to be used in simulations as well as the modelling of edema. MC dose calculations make use of several types of data such as cross-sections, $\frac{\mu_{e n}}{\rho}$ values and tissue compositions to realistically simulate physical processes, and uncertainties in these data contribute to the uncertainty in a MC dose calculation. Tissue compositions are often not well characterized ${ }^{89}$ and uncertainty in the underlying data contributes to the overall uncertainty of the dose calculation. ${ }^{56,90}$

The development of a simulation geometry ${ }^{56}$ to represent the patient also has limitations. Tissue assignment during the creation of VPMs is guided by physiciandrawn organ contours. Contouring is notoriously subjective as it can be difficult to distinguish adjacent substructures, particularly for soft-tisssues. The basis for each initial virtual patient model is a CT image, which does not have very good soft tissue contrast. CT images do, however, clearly display the presence of treatment seeds, but metallic artifacts associated with the seeds can make it difficult to precisely identify the location of a seed. ${ }^{74}$ In addition to the physician drawn contours, the CT images also provide information on the density of the medium in each voxel which can inform the assignment of tissue. However, the density is a continuous variable and it is not obvious what the break-point should be to distinguish one medium from another, and it is possible that this could vary from patient to patient. Furthermore, the composition of the prostate tissue may change as interstitial fluid floods the region, ${ }^{87}$ but it was assumed that the tissue composition was unchanged by edema. The edema modelling procedure is limited to the target only. The motion/deformation of other organs was not included in the expansion model, so dose mapping was restricted to the target volume defined by the physician-drawn contour, which as mentioned above is often an imperfect representation of the actual prostate.

While the effect of edema has been shown to result in global dose reductions in 


\section{CHAPTER 4. DISCUSSION AND CONCLUSIONS}

the target (see figure 3.9) that are as large or larger than the magnitude of reduction from the tissue composition effect (see figure 3.7, 'ref' which shows the ratio of the $\mathrm{D}_{90}$ values from $\mathrm{MC}$ and TG-43 calulations), it is much more difficult to account for edema than tissue composition clinically because observed edema patterns vary considerably between institutions and from patient to patient, ${ }^{19}$ and there is currently no reliable way to predict what kind of edematic response a patient will have before implantation. Whereas a calculation incorporating tissue-based information can be directly calculated if there is a VPM with the appropriate tissue assigned, a dynamic dose calculated with edema must make an assumption for the edema model.

The current understanding of prostatic edema and its mechanisms ${ }^{87}$ are also a limitation. There is no easy adjustment of a PIPB implant post-implant so the inability to predict edema prevents the dosimetric effects of edema from informing a treatment, but a treatment can be evaluated retrospectively. It has been proposed that dynamic dose calculations could be performed post-implant with knowledge of the edema resolution, by taking a series of images post-implant to characterize the edema resolution, and should the treatment be deemed inadequate, the dose to the prostate could be boosted with EBRT. ${ }^{91}$

It has also been proposed that pre-implant shear wave ultrasound elastography to determine the elastic properties of the prostate might prove useful as a tool for predicting an individual patient's susceptibility to edema, ${ }^{45}$ but this has yet to be shown rigorously. As a result of the unpredictability of the edema response there is currently little predictive value of these results in terms of planning for an individual patient, and the wide disparity of different edema responses precludes the selection of an average 'edema correction factor' to be universally applied. However, these results do characterize MC PIPB dose calculation at a population scale, and if a method to predict edema is developed in the future, a solid grounding in the relevant dosimetry principles will allow such data to be usefully included in the planning process. 
In the future, this work should be expanded to incorporate a more complete characterization of the dosimetric effects of edema including a radiobiological analysis. ${ }^{92}$ A finite element modelling approach for generating intermediate VPMs, as described by Mountris et al., ${ }^{45}$ could model deformation of organs outside of the target allowing for meaningful dose evaluation in OARs. Further work could also investigate anisotropic expansions which have been reported. ${ }^{47,93}$

\subsection{Conclusions}

In conclusion, considering the prevalence of prostate cancer and the effectiveness of $\mathrm{PIPB}$, this is a treatment that will see considerable use now and in the future, so improvements to the procedure could translate to tangible benefits for many patients. Two areas where there is room for improvement are accounting for tissue composition and deforming geometries. It was shown here that failing to account for tissue composition leads to an overestimation of $\mathrm{D}_{90}$ by approximately $6 \%$ on average and combined with edema in the aftermath of the PIPB procedure TG-43 dose calculations could overestimate $\mathrm{D}_{90}$ by $20 \%$ or more for a clinically-observed edema model. For accurate dose calculations it is necessary to consider both tissue composition and edema, the latter of which can currently only be evaluated retrospectively after an imaging study. The framework presented and the techniques for calculating accumulated dose are not exclusive to PIPB and could be applied to other edematic organs or indeed any structure that deforms in manner that is spatially and temporally well-defined. 


\section{References}

1 F. Yves, L. Klotz, J. Trachtenberg, and A. Zlotta. The burden of prostate cancer in Canada. Can Urol Assoc J, 3(3 Suppl 2):S92 - S100, 2009.

2 L. Holmberg et al. A randomized trial comparing radical prostatectomy with watchful waiting in early prostate cancer. $N$ Engl J Med, 347(11):781 - 9, 2002.

3 I.F. Tannock et al. Chemotherapy with mitoxantrone plus prednisone or prednisone alone for symptomatic hormone-resistant prostate cancer: a Canadian randomized trial with palliative end points. J. Clin. Oncol., 14(6):1756 - 64, 1996.

4 P. Grimm et al. Comparative analysis of prostate-specific antigen free survival outcomes for patients with low, intermediate and high risk prostate cancer treatment by radical therapy. results from the prostate cancer results study group. BJU International, 109(s1):22-29, 2012.

5 R. Baskar, J. Dai, N. Wenlong, R. Yeo, and K-W. Yeoh. Biological response of cancer cells to radiation treatment. Front Mol Biosci., pages 1-24, 2014.

6 L.A. Moran, H.R. Horton, K.G. Scrimgeour, and M.D. Perry. Principles of Biochemistry (5th Ed). Pearson, Boston, 2012.

7 J.E. Freire, P. De Potter, L.W. Brady, and W.A. Longton. Brachytherapy in primary ocular tumours. Semin. Surg. Oncol., 13(3):167-76, 1997.

8 A.A. Weiner and J.K. Schwarz. Intracavitary brachytherapy for gynecologic malignancies: Applications and innovations. Mo Med, 112(5):366-372, 2015.

9 V. Peppa, E.P. Pappas, P. Karaiskos, T. Major, C. Polgar, and P. Papagiannis. Dosimetric and radiobiological comparison of TG-43 and Monte Carlo calculations in ${ }^{192}$ Ir breast brachytherapy applications. Phys Med., 32(10):1245-1251, 2016.

10 W.F. Whitmore Jr., B. Hilaris, and H. Grabstald. Retropubic implantation to iodine 125 in the treatment of prostate cancer. J. Urol., 108(6):918-20, 1972.

11 ICRU. Report 38: Dose and volume specification for reporting intracavitary therapy in gynecology. $J$ of the ICRU, 20(1), 1985.

12 J.J. Mazeron, P. Scalliet, E. Limbergen, and E. Lartigau. Radiobiology of Brachytherapy and the Dose-Rate Effect in A. Gerbaulet (Ed). The GEC-ESTRO Handbook of Brachytherapy, 012002.

13 E.J. Hall and J. S. Bedford. Dose rate: Its effect on the survival of HeLa cells irradiated with gamma rays. Radiation Research, 22(2):305-315, 1964. 

Report 2010.

M.J. Berger, J.S. Coursey, M.A. Zucker, and J. Chang. ESTAR, PSTAR,
and ASTAR: Computer Programs for Calculating Stopping-power and range ta-
bles for electrons, protons, and helium ions (version 1.2.3), [Online]. Available:
https://physics.nist.gov/Star [2019, June 1]. National Institute of Stan-

M.J. Berger, J.S. Coursey, M.A. Zucker, and J. Chang. ESTAR, PSTAR,
and ASTAR: Computer Programs for Calculating Stopping-power and range ta-
bles for electrons, protons, and helium ions (version 1.2.3), [Online]. Available:
https://physics.nist.gov/Star [2019, June 1]. National Institute of Stan-

M.J. Berger, J.S. Coursey, M.A. Zucker, and J. Chang. ESTAR, PSTAR,
and ASTAR: Computer Programs for Calculating Stopping-power and range ta-
bles for electrons, protons, and helium ions (version 1.2.3), [Online]. Available:
https://physics.nist.gov/Star [2019, June 1]. National Institute of Standards and Technology, Gaithersburg, MD, 2018.

C. Klein et al. Overcoming hypoxia-induced tumor radioresistance in non-small cell lung cancer by targeting DNA-dependent protein kinase in combination with carbon ion irradiation. Radiat Oncol., 12(1):208, 2017.

T.S. Kehwar. Use of Cesium-131 radioactive seeds in prostate permanent implants. J Med Phys, 34:191-193, 2009.

M. Beó, C. Dulieu, X. Mougeot, and M. Kellet. Half-lives: Table of recommended values. Technical Report 2014/18, Laboratoire National Henri Becquerel, Gif-surYvette Cedex, France, 2014.

B.J. Birckhead et al. Stranded seed displacement, migration and loss after permanent implant brachytherapy as estimated by Day 0 fluoroscopy and 4-month postimplant pelvic x-ray. Brachytherapy, 15(6):714-21, 2016.

T. Majoret al. Loose versus stranded seeds in permanent prostate brachytherapy: Dosimetric comparison of intraoperative plans. Physica Medica, 30(8):909-13, 2014.

R. Nath et al. AAPM recommendations on dose prescription and reporting methods for permanent interstitial brachytherapy for prostate cancer: Report of Task Group 137. Med. Phys., 36:5310 - 5322, 2009.

I-125 prostate brachytherapy,. R. Abascal Garcia. Technique of intraoperative planning in prostatic brachytherapy with permanent implants of ${ }^{125} \mathrm{I}$ or ${ }^{103} \mathrm{Pd}$. Arch Esp Urol., 355:1217 - 24, 2002.

E.K. Lee and M. Zaider. Intraoperative dynamic dose optimization in permanent prostate implants. Ann. Thorac. Surg., 56:854-61, 2003.

F. Ballester, R. Nath, and D. Balta. In Jack L.M. Venselaar, Dimos Baltas, Ali S. Meigooni, and Peter J. Hoskin, editors, Comprehensive Brachytherapy: Physical and Clinical Aspects, in Imaging in Medical Diagnosis and Therapy W.R. Hendee, Series Editor, chapter 3, pages 85-103. CRC Press, Boca Raton, Florida, 2012.

M. J. Berger et al. XCOM: Photon cross section database (version 1.5). Technical Report NBSIR87-3597, NIST, Gaithersburg, MD, http://physics.nist.gov/xcom, E. B. Podgorsak. Radiation Physics for Medical Physicists (2nd Ed). SpringerVerlag, Berlin, Heidelberg, 2010. 
R. Nath, L.L. Anderson, G. Luxton, K.A. Weaver, J. F. Williamson, and A. S. Meigooni. Dosimetry of interstitial brachytherapy sources: Recommendations of the AAPM Radiation Therapy Committee Task Group No. 43. Med. Phys., $22: 209-234,1995$.

28 M. J. Rivard et al. Update of AAPM Task Group No. 43 Report: A revised AAPM protocol for brachytherapy dose calculations. Med. Phys., 31:633 - 674, 2004.

29 M. J. Rivard et al. Supplement to the 2004 update of the AAPM Task Group No. 43 Report. Med. Phys., 34:2187 - 2205, 2007.

30 M.J. Rivard et al. Supplement 2 for the 2004 update of the AAPM Task Group No. 43 Report: Joint recommendations by the AAPM and GEC-ESTRO. Med. Phys., 44(9):e297 - e338, 2017.

31 R. G. Stock, N. N. Stone, A. Tabert, C. Iannuzzi, and J. K. DeWyngaert. A doseresponse study for I-125 prostate implants. Int. J. Radiat. Oncol. Biol. Phys., 41:101 - 108, 1998.

32 K Wallner, G Merrick, L True, S Sutlief, W Cavanagh, and W Butler. ${ }^{125}$ I versus ${ }^{103} \mathrm{Pd}$ for low-risk prostate cancer: preliminary PSA outcomes from a prospective randomized multicenter trial. Int. J. Radiat. Oncol. Biol. Phys., 57(5):385-9, 2003.

33 M.A. Kollmeier, R.G. Stock, and N. Stone. Biochemical outcomes after prostate brachytherapy with 5-year minimal follow-up: importance of patient selection and implant quality. Int. J. Radiat. Oncol. Biol. Phys., 57(3):645-53, 2003.

34 L Potters, D Huang, E Calugaru, P Feard, L Lee, and MW Kattan. Importance of implant dosimetry for patients undergoing prostate brachytherapy. Urology, 62(6):1073-7, 2003.

35 B.J. Davis et al. American Brachytherapy Society consensus guidelines for transrectal ultrasound-guided permanent prostate brachytherapy. Brachytherapy, 11(1):6-19, 2012.

36 R. S. Sloboda, N. Usmani, J. Pedersen, A. Murtha, N. Pervez, and D. Yee. Time course of prostatic edema post permanent seed implant determined by magnetic resonance imaging. Brachytherapy, 9(4):354 - 361, 2010.

37 T.T. Monajemi, C.M. Clements, and R.S. Sloboda. Dose calculation for permanent prostate implants incorporating spatially anisotropic linearly time-resolving edema. Med. Phys., 38(4):2289 - 2298, 2011.

38 G. Leclerc, M-C Lavallé, R.C. Roy, E. Vigneault, and L. Beaulieu. Prostatic edema in ${ }^{125} \mathrm{I}$ permanent prostate implants: Dynamical dosimetry taking volume changes into account. Med. Phys., 33(3):574 - 583, 2006. 
F.M. Waterman, N. Yue, B.W. Corn, and A.P. Dicker. Edema associated with I125 or Pd-103 prostate brachytherapy and its impact on post-implant dosimetry: an analysis based on serial CT acquisition. Int. J. Radiat. Oncol. Biol. Phys., 41(5):1069 - 1077, 1998.

40 H. Westendorp, R. Kattevilder, A. van't Riet, A.W. Minken, T.T. Nuver, J.J. Immerzeel, and C.J. Hoekstra. Objective automated assessment of time trends in prostate edema after ${ }^{125}$ I implantation. Brachytherapy, 11:327 - 333, 2012.

41 A. Tejwani et al. Case series analysis of post-brachytherapy prostate edema and its relevance to post-implant dosimetry . J Contemp Brachyther, 4(2):75 - 80, 2012.

42 D. Taussky et al. Sequential evaluation of prostate edema after permanent seed prostate brachytherapy using CT-MRI fusion. Int. J. Radiat. Oncol. Biol. Phys., 62(4):974 - 980, 2005.

43 N. Yue, Z. Chen, R. Peschel, A.P. Dicker, F.M. Waterman, and R. Nath. Optimum timing for image-based evaluation of ${ }^{103} \mathrm{Pd}$ prostate seed implants. Int. J. Radiat. Oncol. Biol. Phys., 45(4):1063 - 1072, 1999.

44 M. Hefny, P. Abolmeasumi, Z. Karimaghaloo, D.G. Gobbi, R. Ellis, and G. Fichtinger. Quantification of of edematic effects in prostate brachytherapy interventions. Med Image Comput Assist Interv., 11(2):493- 500, 2008.

45 K A Mountris et al. Modeling the impact of prostate edema on LDR brachytherapy: a Monte Carlo dosimetry study based on a 3D biphasic finite element biomechanical model. Phys. Med. Biol., 62(6):2087 - 2102, 2017.

Z.J. Chen, J. Deng, K. Roberts, and R. Nath. On the need to compensate for edema-induced dose reductions in preplanned ${ }^{131} \mathrm{Cs}$ Prostate Brachytherapy. Int. J. Radiat. Oncol. Biol. Phys., 70(1):303 - 310, 2007.

47 R.S. Sloboda, N. Usmani, T.T. Monajemi, and D. M. Liu. Impact of edema and seed movements on the dosimetry of prostate seed implants. J Med Phys, 37(2):81 $-89,2012$.

48 J.Z. Wang, N.A. Mayr, S. Nag, J Montebello, and N. Gupta. Effect of edema, relative biological effectiveness, and dose heterogeneity on prostate brachytherapy. Med. Phys., 33(4):1025 - 1032, 2006.

49 T.S. Kehwar, H.A. Jones, M.S. Hug, and R.P. Smith. Influence of prostatic edema on ${ }^{131}$ Cs permanent prostate seed implants: a dosimetric and radiobiological study. Int. J. Radiat. Oncol. Biol. Phys., 80(2):621 - 627, 2011.

50 N. Yue, A.P. Dicker, B.W. Corn, R. Nath, and F.M. Waterman. A dynamic model for the estimation of optimum timing of computed tomography scan for dose evaluation of ${ }^{125} \mathrm{I}$ or ${ }^{103} \mathrm{Pd}$ seed implant of prostate. Int. J. Radiat. Oncol. Biol. Phys., 43(2):447-454, 1997. 
J. Crook, M. McLean, I. Yeung, T. Williams, and G. Lockwood. MRI-CT fusion to assess postbrachytherapy prostate volume and the effects of prolonged edema on dosimetry following transperineal interstitital permanent prostate brachytherapy. Brachytherapy, 3(2):55-60, 2004.

52 A.S. Meigooni, J.A. Meli, and R. Nath. Interseed effects on dose for ${ }^{125} \mathrm{I}$ brachytherapy implants. Med. Phys., 19(2):385-90, 1992.

53 O. Chibani, J.F. Williamson, and D. Todor. Dosimetric effects of seed anisotropy and interseed attenuation for ${ }^{103} \mathrm{Pd}$ and ${ }^{125} \mathrm{I}$ prostate implants. Med. Phys., 32(8):2557-66, 2005.

54 J.F. Carrier and M. D'Amours and F. Verhaegan and B. Reniers and A.G. Martin and E. Vigneault and L. Beaulieu. Postimplant dosimetry using a Monte Carlo dose calculation engine: a new clinical standard. Int. J. Radiat. Oncol. Biol. Phys., 68(4):1190-8, 2007.

55 S. Ramu. Effective atomic numbers for photon energy absorption and photon attneuation of tissues from human organs. Medical Dosimetry, 27(1):1-9, 2002.

56 N. Miksys, E. Vigneault, A-G Martin, L. Beaulieu, and R.M. Thomson. Largescale retrospective Monte Carlo dosimetric study for permanent implant prostate brachytherapy. Int. J. Radiat. Oncol. Biol. Phys., 97(3):606-615, 2017.

57 A. C. Collins Fekete, M. Plamondon, A.G. Martin, E. Vigneault, F. Verhaegen, and L. Beaulieu. Calcifications in low-dose rate prostate seed brachytherapy treatment: post-planning dosimetry and predictive factors. Radiother. Oncol., 114(3):339 - 44, 2015.

58 O. Chibani and J.F. Williamson. MCPI: a sub-minute Monte Carlo dose calculation engine for prostate implants. Med. Phys., 32(12):3688-98, 2005.

59 M Chamberland, R E P Taylor, D W O Rogers, and R M Thomson. egs_brachy: a versatile and fast Monte Carlo code for brachytherapy. Phys. Med. Biol., 61:8214 - 8231, 2016.

60 P. Papagiannis, E. Pantelis, and P. Karaiskos. Current state of the art brachytherapy treatment planning dosimetry algorithms. Brit. J. Radiol., 87(1041):20140163, 2014.

61 K.A. Gifford, J.L. Horton, T.A. Wareing, G. Failla, and F. Mourtada. Comparison of a finite-element multigroup discrete-ordinates code with monte carlo for radiotherapy calculations. Phys Med Biol., 51(9):2253 - 65, 2006.

62 A.K. Carlsson and A. Ahnesjö. The collapsed cone superposition algorithm applied to scatter dose calculations in brachytherapy. Med. Phys., 27:2320 - 2332, 2000 .

63 A.C. Collins-Fekete, M. Plamondon, A.G. Martin, E. Vigneault, F. Verhaegen, and L. Beaulieu. Quantifying the effect of seed orientation in postplanning dosimetry of low-dose-rate prostate brachytherapy. Med. Phys., 41(10):101704-1 $-101704-9$ year $=2014$, 

of tissue heterogeneity for ${ }^{125}$ I prostate implants. Reports of Practical Oncology and Radiotherapy, 19:392 - 398, 2014.

65 NCRP. Report No. 58: A Handbook of Radioactivity Measurements Procedures, 2nd ed. National Council on Radiation Protection and Measurements, Bethesda, MD, 1985.

66 M. Fippel. In Joao Saco and Frank Verhaegen, editors, Monte Carlo Techniques in Radiation Therapy, Imaging in Medical Diagnosis and Therapy W.R. Hendee, Series Editor, chapter 3, pages 29-43. CRC Press, Boca Raton, Florida, 2013.

67 J.F. Williamson. Monte Carlo evaluation of kerma at a point for photon transport problems. Med. Phys., 14(5):567-576, 1987.

68 K.A. Kovtun et al. Dosimetry quality and evolution of edema after low-doserate brachytherapy for small prostates: Implications for the use of new isotopes. Brachytherapy, 13:152 - 6, 2014.

69 O. Tanaka et al. Effect of edema on postimplant dosimetry in prostate brachytherapy using CT/MRI fusion. Int. J. Radiat. Oncol. Biol. Phys., 69(2):614 - 618, 2007.

70 F.M. Waterman and A.P. Dicker. Impact of postimplant edema on V100 and D90 in prostate brachytherapy: Can implant quality be predicted on day 0? Int. J. Radiat. Oncol. Biol. Phys., 53(3):610 - 621, 2002.

71 J.V. Siebers and H. Zhong. An energy transfer method for 4D Monte Carlo dose calculations. Med. Phys., 35(9):4096-4105, 2008.

72 H.S. Li, H. Zhong, J. Kim, C. Glide-Hurst, M. Gulam, T.S. Nurushev, and I.J. Chetty. Direct dose mapping versus energy/mass transfer mapping for 4D dose accumulation: fundamental differences and dosimetric consequences. Phys. Med. Biol., 59:173-188, 2014.

73 E. Heath, F. Tessier, and I. Kawrakow. Investigation of voxel warping and energy mapping approaches for fast 4D Monte Carlo dose calculations in deformed geometries using VMC++. Phys. Med. Biol., 56(16):5187 - 5202, 2011.

74 N. Miksys, C. Xu, L. Beaulieu, and R. M. Thomson. Development of virtual patient models for permanent implant brachytherapy Monte Carlo dose calculations: interdependence of CT image artifact mitigation and tissue assignment. Phys. Med. Biol., 60(15):6039, 2015.

75 R.M. Thomson, R.E.P. Taylor, and D. W. O. Rogers. Monte Carlo dosimetry for ${ }^{125} \mathrm{I}$ and ${ }^{103} \mathrm{Pd}$ eye plaque brachytherapy. Med. Phys., 35(12):5530-5543, 2008.

76 Y. Ma et al. A generic TG-186 shielded applicator for commissioning model-based dose calculation algorithms for high-dose-rate ${ }^{192} \mathrm{Ir}$ brachytherapy. Med. Phys., 44:5961 - 5976, 2017. 
77 R.M. Thomson, R.E.P. Taylor, M.J.P. Chamberland, and D.W.O. Rogers. Reply to Comment on 'egs_brachy: a versatile and fast Monte Carlo code for brachytherapy'. Phys. Med. Biol., 63(3):038002(5pp), 2018.

M. Rodriguez and D. W. O. Rogers. On determining dose rate constants spectroscopically. Med. Phys., 40:011713 (10pp), 2013.

I. Sechopoulos et al. RECORDS: improved Reporting of montE CarlO RaDiation transport Studies: Report of AAPM Research Committee TG 268. Med. Phys., 45(1):e1-e5, 2018.

R.E.P Taylor and D.W.O Rogers. An EGSnrc Monte Carlo-calculated database of TG-43 parameters. Med. Phys., 35:4228 -4241, 2018.

81 I. Kawrakow and M. Fippel. Investigation of variance reduction techniques for Monte Carlo photon dose calculation using XVMC. Physics in Medicine and Biology, 45(8):2163-2183, 2000.

82 M. Martinov and R.M. Thomson. User Guide for 3ddose_tools v1.1. Carleton Laboratory for Radiotherapy Physics, Ottawa, Ontario, 2016.

83 R.M. Thomson, R.E.P. Taylor, M.J.P. Chamberland, and D.W.O. Rogers. User manual for egs_brachy: A versatile and fast EGSnrc application for brachytherapy. Carleton Laboratory for Radiotherapy Physics, Ottawa, Ontario, 2017.

84 M. Rosu, I.J. Chetty, J.M. Balter, M.L. Kessler, D.L. McShan, and R.K. Ten Haken. Dose reconstruction in deforming lung anatomy: Dose grid size effects and clinical implications. Med. Phys., 32(8):2487-2495, 2005.

85 MATLAB. version 7.10 .0 (R2010a). The MathWorks Inc., Natick, Massachusetts, 2010.

86 Blender Online Community. Blender 2.78 - a 3D modelling and rendering package. Blender Foundation, Blender Institute, Amsterdam, 2016.

87 J. Scallan, V.H. Huxley, and R.J. Korthuis. Capillary Fluid Exchange: Regulation, Functions and Pathology. Morgan \& Claypool Life Sciences, San Rafael, California, 2010.

88 Z.J. Chen, J.D. Deng, K. Roberts, and R. Nath. Potential impact of prostate edema on the dosimetry of permanent seed implants using the new ${ }^{131} \mathrm{Cs}$ (model CS-1) seeds. Med. Phys., 33(4):968-975, 2006.

89 D. Mann-Krzisnik, F. Verhaegen, and S.A. Enger. The influence of tissue composition uncertainty on dose distributions in brachytherapy. Radiother Oncol, 126(3):394-410, 2018.

90 C. Villagrasa et al. Assessing the contribution of cross-sections to the uncertainty of Monte Carlo calculations in micro- and nanodosimetry. Radiat Prot Dosimetry, 183(2):11-16, 2018. 
91 N. Yue, J. Mori, R. Nath, D.E. Heron, and M.S. Huq. External beam radiotherapy boosts to reduce the impact caused by edema in prostate permanent seed implants. Physics in Medicine and Biology, 51(9):2267-2277, 2006.

92 N. Miksys, M. Haidari, E. Vigneault, A-G Martin, L. Beaulieu, and R.M. Thomson. Coupling I-125 permanent implant prostate brachytherapy Monte Carlo dose calculations with radiobiological models. Med. Phys., 44:4329-4340, 2017.

93 R. Nakamura et al. Effects of ellipsoid prostate deformation on dose delivery during permanent interstitial brachytherapy. Brachytherapy, 35(10):208-213, 2011. 